\title{
Structure of Molecules by NMR Spectroscopy Using Liquid Crystal Solvents
}

\section{N. SURYAPRAKASH}

Sophisticated Instruments Facility, Indian Institute of Science, Bangalore-560 012, India; Phone: 91-80-309-2536; Fax: 91-80-334-1683; E-mail: nsp@sif.iisc.ernet.in

\begin{abstract}
Nuclear magnetic resonance spectroscopy of molecules aligned in nematic liquid crystalline media provides information on the molecular structure and order parameters. The partial alignment of the molecules in the liquid crystalline phases gives rise to residual intramolecular dipolar couplings which are dependent on internuclear distances, and therefore yield information on the structure of molecules. The dipolar couplings are in general large compared to chemical shifts, and the spectra become rapidly complex with the increase in the number of interacting spins and decrease of symmetry of molecules. In this article, the procedure for the analyses of such spectra is outlined in a pedagogical manner. The concept of the order parameter is illustrated in a phenomenological way. The utility of the technique and obtainable information is demonstrated using practical examples.
\end{abstract}

KEY WORDS: liquid crystals; director; nematic phase; oriented molecules; order parameter; dipolar couplings; computer programs; root mean square error; internuclear distance; molecular structure; vibrational correction

\section{INTRODUCTION}

Nuclear magnetic resonance (NMR) spectroscopy provides information on molecular structure, conformation, and dynamics. This information can be derived from such parameters as chemical shifts $\left(\delta_{i}\right.$ 's), indirect spin-spin couplings $\left(J_{i j}\right.$ 's), direct dipolar couplings $\left(D_{i j}\right.$ 's), spin-lattice relaxation times $\left(T_{1}\right.$ 's) and spin-spin relaxation times $\left(T_{2}\right.$ 's $)$ (1).

The line positions and line intensities in the high-resolution NMR spectra of diamagnetic materials in liquid or solution states depend only on chemical shifts and indirect spin-spin couplings, and information on the molecular structure is obtained using these parameters. The dipolar couplings arise because of the through-space interaction of nuclear dipoles, and for most of the molecules this interaction vanishes in the liquid state at the magnetic field strengths of several tesla normally employed, because of the rapid isotropic tumbling motion of the molecules. This is because the dipolar interaction is proportional to $\left(3 \cos ^{2} \theta_{i j}-1\right) / r_{i j}^{3}$, where $r_{i j}$ is the distance between the nuclei $i$ and $j$ and $\theta_{i j}$ is the angle this internuclear vector makes with respect to the magnetic field direction that vanishes for rapid isotropic molecular orientations. One advantage of such an averaging process is that it results in simplified spectra; the disadvantage is that the dipolar information is lost and does not give 
additional information on the molecular geometry. In solids, both intra- and intermolecular dipolar couplings are present, resulting in broad and featureless spectra. The dipolar coupling information is usually extracted from the line-width measurements, and hence the derived information on the molecular geometry is less precise.

In an intermediate state where there is sufficient translational mobility between molecules, the intermolecular dipolar couplings are averaged to zero. If at the same time there are molecular orientations that are not equally probable resulting in a restricted ordering, then the averaged intramolecular dipolar couplings are nonzero. In such cases, it is possible to get well-resolved spectra containing dipolar coupling contributions, which in turn provide geometry information with high precision.

Such an intermediate phase between solids and liquids can be realized by dissolving the solute molecule to be studied in a liquid crystalline solvent. A typical example of the difference in the appearance of NMR spectra of an organic molecule in liquid, liquid crystalline, and solid phases is shown in Fig. 1.

The object of this article was to provide a pedagogical introduction to liquid crystals and the analyses of the NMR spectra of molecules oriented in such phases. Further, interpretation of the dipolar couplings in terms of structure and orientation of the molecules is discussed using practical examples. However, discussion of this technique is restricted to spin- $\frac{1}{2}$ nuclei in this article.

\section{LIQUID CRYSTALS}

Materials that exhibit liquid crystallinity combine the flow properties of liquids and the optical properties, such as birefringence, of crystals (2-4). The liquid crystals are broadly classified into two types, viz., thermotropic and lyotropic. In thermotropic liquid crystals (generally materials with elongated molecules), the liquid crystalline phase, also called the mesophase, arises over a well-defined range of temperatures.

The thermotropic liquid crystals are further classified as nematic, smectic, and cholesteric, depending on the arrangement of molecules and the degree of order. In nematics, molecules are preferentially aligned such that their long axes are arranged parallel to each other. The preferred orientation of the long axes of the mol-
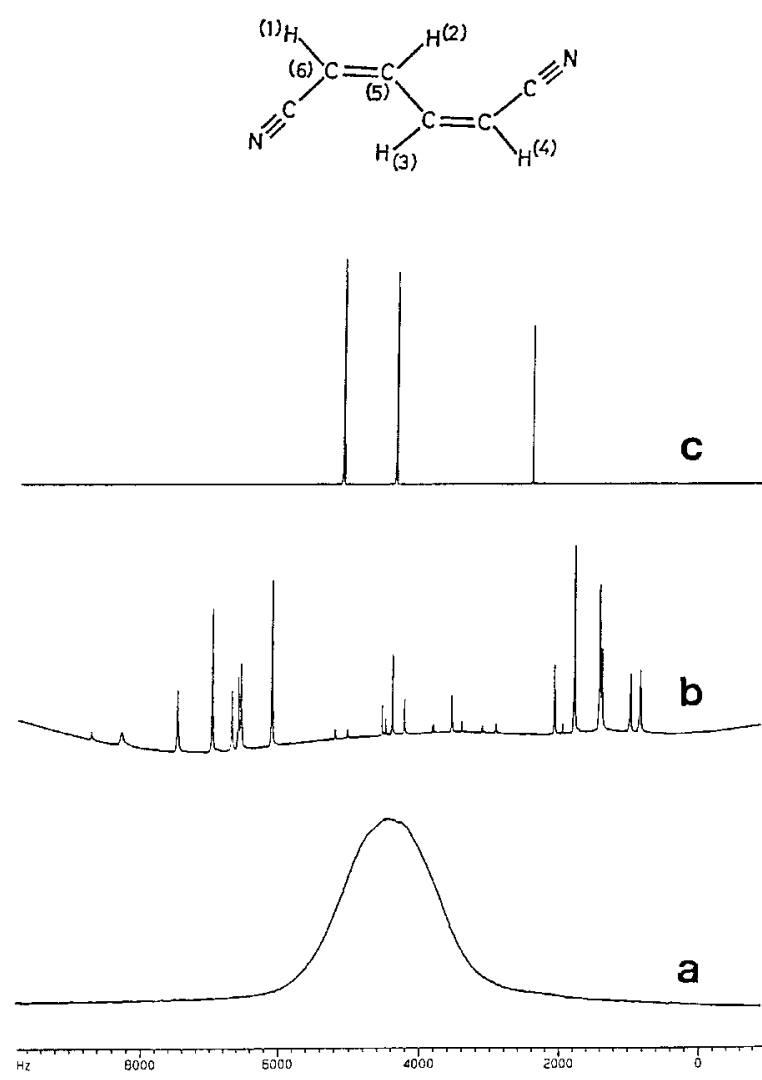

Figure 1 Proton NMR spectra of cis,cis-mucononitrile in (a) pure solute in the solid state, (b) dissolved (6 $\mathrm{wt} \%$ ) in the liquid crystal ZLI-1114, and (c) isotropic solution state in the solvent $\mathrm{CDCl}_{3}$. All spectra were recorded at ambient temperature on a Bruker DRX-500 NMR spectrometer under identical conditions. Note in this unusual example that the width of the solute in the solid state is lesser than in the anisotropic medium.

ecules is generally referred to by a unit vector called the "director," usually denoted as $\hat{n}$. In smectics, molecules are arranged parallel to each other and are separated into layers such that their long axes preferentially point in the same direction. Smectics are further classified into smectic A, smectic B, smectic C, etc., depending on the molecular arrangement within and between the layers. Cholesterics are special examples of nematics and are formed by optically active molecules. Molecules in cholesterics are arranged parallel to each other with a continuous displacement of the director such that the overall displacement follows a helical path. An ideal view of different thermotropic liquid crystal phases (4) is shown in Fig. 2.

Lyotropic liquid crystals are obtained by a mixture of two or more components, one of which is amphiphilic and the other which is generally po- 
(a)

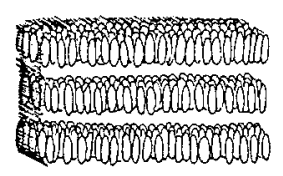

(c)

(b)

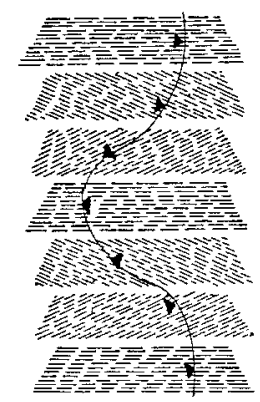

Figure 2 Ideal view of different types of liquid crystals: (a) nematic, (b) cholesterics, (c) smectic A, (d) smectic B, and (e) smectic C.

lar (usually water) (5). In some cases, nonpolar organic solvents such as alkanes are used instead of water. Amphiphilic compounds have two distinct groups: hydrophilic and lipophilic. The hydrophilic group of the molecule is soluble in a polar solvent such as water. The lipophilic group of the molecule is soluble in nonpolar solvents. Since most times water is used as a polar solvent in lyotropics, lipophilic groups are generally referred to as hydrophobic. Depending on the hydrophobic and hydrophilic parts, the amphiphilic compounds exhibit remarkable solubility properties. They can be predominantly hydrophilic - that is, water soluble and hydrocarbon insoluble-or predominantly lipophilic - that is, water insoluble and hydrocarbon soluble.

At extreme dilution, the amphiphilic compounds are distributed randomly throughout the solution. As the concentration of the amphiphilic molecules is increased, molecules start aggregating. At a concentration known as critical micelle concentration, aggregates of the molecules begins to form stable entities known as micelles. In the micellar form, the nonpolar hydrocarbon chains occupy the interior of the micelles and the polar head groups occupy the outer periphery of the aggregates. It is very interesting to note that the head groups are placed in such close association that the hydrophobic part is completely out of contact with water. The micelles do not have a fixed shape. They are statistical in nature. They fluctuate constantly in size and shape in response to temperature and concentration.

A very good example of this lyotropic phase is common soap. Common soap is sodium dodecylsulphate, where sulphate is the head group and the hydrocarbon chain with 12 carbon atoms forms a tail group.

With different proportions of amphiphilic compounds in water, a variety of lyotropic liquid crystal phases are observed and are accordingly classified depending on the alignment of molecules in these phases. Details of the various lyotropic phases are available in the literature $(5,6)$.

\section{Choice of Liquid Crystal as a Solvent}

Lyotropic liquid crystals serve as appropriate solvents generally for the study of polar molecules and ions. For other systems, work is usually undertaken in thermotropic liquid crystals. Our discussion in the remaining part of this article is restricted to thermotropic liquid crystals.

Of the three different types of thermotropic liquid crystals, nematics are the least ordered and generally precede the isotropic transition temperature. Thus, nematics, because of high mobility, are more commonly used as solvents in NMR studies. Smectic phases are rarely used. The reason for this may be due to their very high viscosity. Cholesterics also are not routinely used as solvents in NMR studies, probably owing to difficulty in their alignment. On the other hand, studies using cholesterics, however, may provide information on the optically active solutes (7-10).

There are several thermotropic liquid crystals available for NMR studies. The molecular structures and transition temperatures of a few typical thermotropic nematics commonly used as solvents in NMR are given in Fig. 3. However, the choice of the liquid crystal for a particular study depends on the solubility of the solute.

\section{Solute Molecules in a Nematic Phase}

A nematic phase has a long-range orientational order. That is, the long axes of the molecules tend to align parallel to each other. The orientations of the long axes of all the molecules are not the same. Hence, the ensemble average of all possible alignments of the long axes is not zero, and there is a preferential orientation along a particular direction. This phenomenon causes the liquid crystal molecules to exhibit an orientational or- 


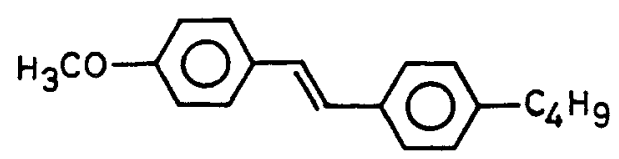

(a)

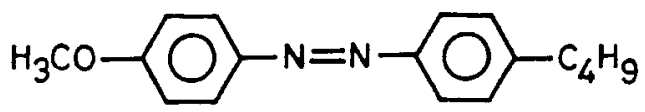

(b)

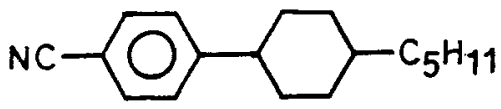

(c)

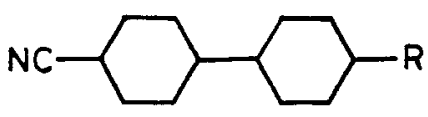

(d)

$$
\mathrm{R}=\mathrm{C}_{3} \mathrm{H}_{7}, \mathrm{C}_{5} \mathrm{H}_{41}, \mathrm{C}_{7} \mathrm{H}_{15}
$$

Figure 3 Some commonly used nematic liquid crystals and their mesophase ranges: (a) $N$ - $p$-methoxybenzylidene)- $p^{\prime}-n$-butylaniline (MBBA), 293-321 K; (b) $p$ $n$-butyl- $p$ '-methoxyazoxybenzene (phase IV), 289-349 K; (c) trans-pentyl-(4-cyanophenyl)-cyclohexane (S-1114), 303-328 K; and (d) a ternary eutectic mixture of propyl, pentyl, and heptyl bicyclohexyl carbonitrile (ZLI-1167), 281-356 K.

der. Furthermore, nematics generally exhibit cylindrical symmetry along the director. Because of this, measurement of any property of this phase - such as magnetic susceptibility, polarizability, etc. - will exhibit one value parallel to the symmetry axis and another value in all directions perpendicular to the symmetry axis. For example, if the magnetic susceptibility $(\chi)$ is measured, along $\left(\chi_{\|}\right)$and orthogonal $\left(\chi_{\perp}\right)$ to the symmetry axis, then $\Delta \chi\left(=\chi_{\|}-\chi_{\perp}\right)$ is finite. Thus, the bulk properties of these molecules are anisotropic. At the microscopic level, these molecules are mobile, and the mobility can be compared to that of a liquid. That is, the centers of mass of the molecules are distributed at random throughout the volume. In other words, these molecules do not have any translational order.

For a small solute molecule dissolved in a nematic phase, the anisotropic environment of the liquid crystal provides an orientational order and a high degree of mobility, i.e., a translational disorder. Consequences of these phenomena are the presence of finite intramolecular dipolar cou- plings between the spins of molecules and the averaging of all intermolecular dipolar couplings to zero. The proton NMR spectrum of such a sample then generally consists of sharp lines arising from dissolved, relatively small molecules riding over a broad background of a large number of resonances arising from the liquid crystal protons. The sharp lines obtained from the solutes in nematics result from the orientation of the director in the magnetic field. However, it should be pointed out that not all liquid crystals align in the magnetic field. In such cases, broad lines are observed. An example could be a smectic A liquid crystal possessing a high-temperature nematic. Initially, the solute molecules give rise to broad lines. When the sample is heated and cooled to the nematic phase, keeping the sample in the magnet results in sharp lines for the dissolved solute molecule. Furthermore, in general, the degree of order of the solvent is high and that of the solute is low. Thus, the spectra of the solute clearly separates out into sharp, well-resolved lines from the background of the solvent. However, in the unusual example, the dissolved solute molecule exhibits high order and its magnitude is comparable to that of a solvent (11). Even in such a case, sharp lines are observed. This fundamental difference of getting well-resolved lines dominated by dipolar couplings, compared to solids and liquids, is the basis of the NMR spectroscopy of molecules aligned in liquid crystals.

\section{HAMILTONIAN OF THE ORIENTED SYSTEM}

To understand how dipolar couplings are manifested in the NMR spectrum of oriented systems, let us look at the basic Hamiltonian. For spin- $\frac{1}{2}$ nuclei, the Hamiltonian governing the NMR spectra of aligned molecules is given by (12):

$$
\begin{aligned}
H= & -\sum_{i}\left(1-\sigma_{i}-\sigma_{i}^{a}\right) v_{o} I_{z i} \\
& +\sum_{i<j} \sum_{i j}\left(J_{i j} D_{i j}(\operatorname{dir})\right) I_{z i} I_{z j} \\
& +(1 / 2) \sum_{i<j}\left(J_{i j}-D_{i j}(\operatorname{dir})\right)\left(I_{i}^{+} I_{j}^{-}+I_{i}^{-} I_{j}^{+}\right)
\end{aligned}
$$

In the above equation, $J_{i j}$ and $\sigma_{i}$ represent one third of the traces of the corresponding tensors 
and are identical to the indirect spin-spin couplings and chemical shifts observed in the highresolution NMR in the isotropic media. $\sigma_{i}^{a}$ correspond to the shielding constants observed in the anisotropic media. $D_{i j}($ dir $)$ is the direct dipolar couplings. The Hamiltonian for the oriented systems differs from that for the isotropic systems in that there are additional terms, viz., chemical shielding due to anisotropic environment and the dipolar couplings that are finite in the anisotropic media. Thus, in the above Hamiltonian, the substitution of $\left(1-\sigma_{i}\right)$ for $\left(1-\sigma_{i}-\sigma_{i}^{a}\right)$ and $J_{i j}$ for $\left(J_{i j}+2 D_{i j}(\mathrm{dir})\right)$ and $\left(J_{i j}-D_{i j}(\mathrm{dir})\right)$ provides the total Hamiltonian for the isotropic system. $J_{i j}$ will also have the anisotropic part and contribute significantly in some cases. However, of the various parameters in the anisotropic media, the dipolar interaction is of much more relevance in the structure determination.

\section{Dipolar Couplings}

The classical interaction energy $E$ between two magnetic dipole moments $\mu_{1}$ and $\mu_{2}$ is given by

$$
E=\frac{\mu_{1} \cdot \mu_{2}}{r^{3}}-\frac{3\left(\mu_{1} \cdot r\right)\left(\mu_{2} \cdot r\right)}{r^{5}}
$$

where $r$ is the radius vector from $\mu_{1}$ to $\mu_{2}$.

Treating $\mu_{1}$ and $\mu_{2}$ as operators, i.e., $\mu_{1}=$ $\gamma_{\mathrm{I}} \hbar I_{1}$ and $\mu_{2}=\gamma_{\mathrm{I}} \hbar I_{2}$ and expressing the interaction energy of the magnetic dipoles in polar coordinates, the dipolar Hamiltonian can be explicitly written as

$$
\begin{aligned}
H_{D}= & \left(\gamma_{i} \gamma_{j} h / 4 \pi^{2}\right) 1 / r_{i j}^{3} \\
& \cdot[A+B+C+D+E+F]
\end{aligned}
$$

where

$$
\begin{gathered}
A=\left(1-3 \cos ^{2} \theta_{i j}\right) \cdot I_{Z i} \cdot I_{Z j} ; \\
B=-1 / 4\left(1-3 \cos ^{2} \theta_{i j}\right) \cdot\left[I_{i}^{+} I_{j}^{-}+I_{i}^{-} I_{j}^{+}\right] ; \\
C=-3 / 2 \sin \theta_{i j} \cos \theta_{i j} e^{-i \phi}\left(I_{i}^{+} I_{Z j}+I_{Z i} I_{j}^{+}\right) ; \\
D=C^{*} ; \\
E=-3 / 4 \sin ^{2} \theta_{i j} e^{-2 i \phi} I_{i}^{+} I_{j}^{+} ; \text {and } \\
F=E^{*} .
\end{gathered}
$$

Terms $C$ and $D$ produce transitions at $\omega_{0}$, and $E$ and $F$ produce transitions at $2 \omega_{0}$ which are otherwise forbidden. With the increasing strength of the magnetic field $H_{0}$, the contributions by $C$, $D, E$, and $F$ terms become weaker. In the high field approximation (several tesla of the magnetic field normally used in NMR studies), the contribution from the terms $C-F$ to $H_{D}$ becomes negligible. These terms can therefore be dropped from $H_{D}$. Under this approximation, known as secular approximation, only the terms $A$ and $B$ are thus important and contribute to the NMR spectrum. However, it is to be remembered that the terms $C-F$ are important for relaxation. The dipolar Hamiltonian can then be written as

$$
\begin{gathered}
H_{D}=\left(\gamma_{i} \gamma_{j} h / 4 \pi^{2}\right) 1 / r_{i j}^{3}[A+B] \\
H_{D}=\left(\gamma_{i} \gamma_{j} h / 4 \pi^{2}\right)\left(1-3 \cos ^{2} \theta_{i j}\right) / r_{i j}^{3} \\
\cdot\left[I_{z i} \cdot I_{z j}-1 / 4\left(I_{i}^{+} I_{j}^{-}+I_{i}^{-} I_{j}^{+}\right)\right]
\end{gathered}
$$

The spatial part of the dipolar Hamiltonian partially averaged in a liquid crystalline phase can be written as

$$
D_{i j}(\operatorname{dir})=\frac{-\left(\gamma_{i} \gamma_{j} h\right)}{4 \pi^{2}} \frac{1}{2}\left\langle\frac{3 \cos ^{2} \theta_{i j}-1}{r_{i j}^{3}}\right\rangle
$$

The quantity within the angular brackets is the average taken over all molecular motions. For a given pair of nuclei, $\left(\gamma_{i} \gamma_{j} h / 4 \pi^{2}\right)$ is a constant and can be denoted as $K_{i j}$. Then, Eq. [6] can be rewritten as

$$
D_{i j}(\operatorname{dir})=-K_{i j} \frac{1}{2}\left\langle\frac{3 \cos ^{2} \theta_{i j}-1}{r_{i j}^{3}}\right\rangle
$$

If there is no correlation between the internuclear distance $r_{i j}$ and the term $\left\langle 3 \cos ^{2} \theta_{i j}-1\right\rangle$, Eq. [7] becomes

$$
D_{i j}(\mathrm{dir})=-K_{i j}(1 / 2)\left\langle 3 \cos ^{2} \theta_{i j}-1\right\rangle\left\langle 1 / r_{i j}^{3}\right\rangle
$$

For rigid molecules,

$$
D_{i j}(\operatorname{dir})=-\left(K_{i j} / r_{i j}^{3}\right)(1 / 2)\left\langle 3 \cos ^{2} \theta_{i j}-1\right\rangle
$$

In a short form,

$$
D_{i j}(\operatorname{dir})=-K_{i j} \cdot S_{i j} / r_{i j}^{3}
$$

where $S_{i j}=(1 / 2)\left\langle 3 \cos ^{2} \theta_{i j}-1\right\rangle$ is referred to as the orientation or order parameter [13]. It is implicit from Eq. [10] that knowledge of the dipolar couplings provides information on the geomet- 
rical as well as the orientational parameters of the molecules in a liquid crystalline medium. The order parameter is discussed conceptually using the molecular theory of liquid crystals (14) in the next section.

Equation [10] further suggests that the interaction Hamiltonian contains the ratio of $S_{i j}$ to $r_{i j}^{3}$. $D_{i j}$ (dir)'s remain invariant by the multiplication of the numerator and the denominator of Eq. [10] by a constant factor. Experimental measurements can, therefore provide only this ratio. It follows that this method provides relative values of internuclear distances and not absolute values. However, the absolute values of bond angles can be determined. Hence, this method provides only the shape of the molecules, and not the size.

\section{Anisotropy of the Indirect Spin - Spin Couplings $\left(J_{i i}^{a}\right)$}

The experimentally determined dipolar couplings are the sum of the dipolar coupling and the anisotropic contribution of the indirect spin-spin couplings $\left(J_{i j}^{a}\right)$. The multiplier of the spin operator for $\left(J_{i j}^{a}\right)$ has the same form as that of the dipolar coupling given in Eq. [5], and therefore, $J_{i j}^{a}$ is also called pseudo-dipolar coupling. The dipolar couplings obtained from the NMR spectra [ $D_{i j}\left(\right.$ expt)] are therefore $D_{i j}(\operatorname{dir})+J_{i j}^{a}$. For a pair of protons, $J_{i j}^{a}$ is usually negligible, since the major contribution to $J_{i j}^{a}$ comes from the spherically symmetric Fermi contact term. In such cases, the measured direct dipolar couplings immediately provide the geometry information. For heavy nuclei such as fluorine, $J_{i j}^{a}$ may, however, be significant.

\section{ORDER PARAMETER(S)}

\section{Phenomenological Concept of Nematic Order $(14,15)$}

We can now define the order parameter, which is nonzero in the nematic phase and vanishes in the isotropic phase. The identification of the appropriate order parameter for nematic liquid crystals is aided by a consideration of the observed structure and symmetry of the phase. The nematic phase usually exhibits cylindrical symmetry. We can denote this cylindrical symmetry axis as the director. The anisotropic property of the nematics arises because of the tendency of the rodlike molecules to align with their long axes parallel to

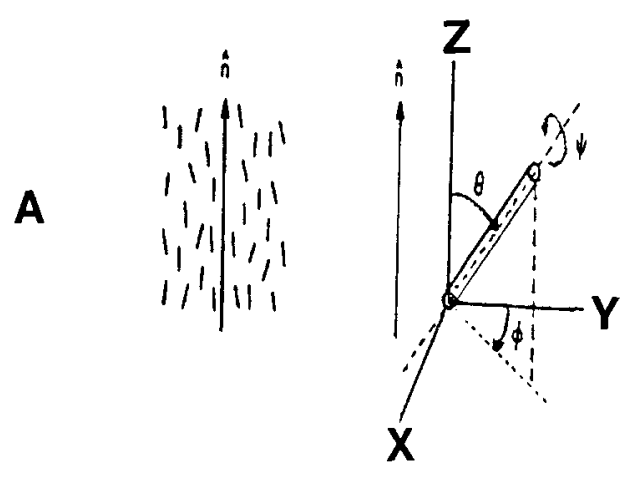

B

Figure 4 Schematic showing the orientation of a molecule in a nematic liquid crystal: (A) orientation of the director ( $\hat{\mathrm{n}}$ ), chosen to be along the $Z$ axis, and (B) Eulerian angles required to describe the orientation.

the director, as shown in Fig. 4(A). At finite temperatures, the thermal motion of the molecules prevents perfect alignment with the director, and the orientations of the molecules are in fact distributed.

Let us look at the orientation of any single molecule with respect to the director. Let the director lie along the $Z$-axis of a fixed rectangular coordinate system. The orientation of the rodlike molecule can then be described using three Eulerian angles, i.e., $\theta, \phi$, and $\psi$, as shown in Fig. 4(B). Here, $\psi$ and $\phi$ correspond to the rotation of the molecule along the axis of the cylinder and the azimuthal direction, respectively.

Because of cylindrical symmetry, there is no ordering in the angles $\phi$ and $\psi$. Thus, we are left with only the parameter $\theta$ for which a degree of order can exist. When all alignments of the molecules are considered, the state of alignments of the molecules can be described by a function of $\theta$, i.e., $f(\theta)(15)$. The simplest function one can then think of is to take the average of the projection of all molecules along the director, i.e., $\langle\cos \theta\rangle$. However, the integral of this function vanishes over all possible values of $\theta$, because $f(\theta)=f(\pi-\theta)$. Thus, we must resort to higher orders of this function. The first such function giving a nontrivial answer is $\left\langle\cos ^{2} \theta\right\rangle$. For the situation when $\theta=0$, all molecules are fully aligned along the director; thus,

$$
S=\left\langle\cos ^{2} \theta\right\rangle=1
$$

If the orientation is entirely random, then all values of $\theta$ are equally likely and

$$
S=\left\langle\cos ^{2} \theta\right\rangle=1 / 3
$$


In any order-disorder problem, the convention followed is that the maximum value of the order is taken to be 1 when the system is completely ordered, and zero when the system is disordered. To satisfy these two conditions, Eqs. [11] and [12] can be combined as

$$
S=1 / 2\left(\left\langle 3 \cos ^{2} \theta-1\right\rangle\right)
$$

This gives $S=1$ and -0.5 for the preferential alignment of the molecules in the direction parallel and perpendicular to the director, respectively. For any intermediate orientation, $S$ varies between 1 and $-0.5 . S$ is therefore a measure of the order.

The orientation of the director of the liquid crystal in the magnetic field depends on the sign of the diamagnetic susceptibility anisotropy $(\Delta \chi)$ of the liquid crystal. If $\Delta \chi$ is positive, the director aligns parallel to the magnetic field, and it is orthogonal to the magnetic field if $\Delta \chi$ is negative.

\section{The Order Parameter of a Molecule Aligned in a Nematic Phase}

When the solute molecule is dissolved in a nematic phase, it will be aligned by the neighboring nematic molecules, and this alignment will also be characterized by an order parameter $S$. For any rigid molecule of an arbitrary shape, if nuclei $i$ and $j$ belong to the same rigid part of the molecule, the order parameter can be described by a $3 \times 3$ matrix, $S_{i j}$. Let us assume that the director of the liquid crystal is parallel to the magnetic field direction, and $X, Y$, and $Z$ are the molecule fixed coordinate system. If $\theta_{X}, \theta_{Y}$, and $\theta_{Z}$ are the angles between the molecular axes and the magnetic field direction, then the definition of the ordering matrix elements is given by

$$
\begin{array}{r}
S_{a b}=(1 / 2)\left\langle 3 \cos \theta_{a} \cos \theta_{b}-\delta_{a b}\right\rangle \\
a, b=x, y, z
\end{array}
$$

where $\delta_{a b}$ is the kronecker delta $\left(\delta_{a b}=1\right.$ for $a=b$ and 0 for $a \neq b$ ). The matrix $S_{a b}$ of Eq. [14] is explicitly written as

$$
\left|\begin{array}{lll}
S_{X X} & S_{X Y} & S_{X Z} \\
S_{Y X} & S_{Y Y} & S_{Y Z} \\
S_{Z X} & S_{Z Y} & S_{Z Z}
\end{array}\right|
$$

This ordering matrix is symmetric and traceless and has five independent elements, viz., $S_{X X}$, $S_{Y Y}, S_{X Y}, S_{X Z}, S_{Y Z}$.

\section{Order Parameter and Molecular Symmetry}

The $S_{i j}$ values of different molecular axes are interdependent. Depending on the symmetry of the molecule and the suitable choice of the coordinate system, the number of independent elements of the ordering matrix required to describe molecular orientation varies from zero to five. A table of the number of independent nonzero elements of the ordering matrix that describes the orientation of the molecules with different point group symmetry is available in the literature [4]. A few examples of molecules with different symmetry are described below.

Molecules with tetrahedral or cubic symmetry do not orient ( $S$ value is zero) even in an anisotropic environment, and hence, the dipolar couplings in such systems average to zero. However, in actual practice, there are minor distortions in the symmetry and dipolar splittings are observed. In the example of the methane molecule shown in Fig. 5(A), splittings are observed $(16,17)$. Spec-
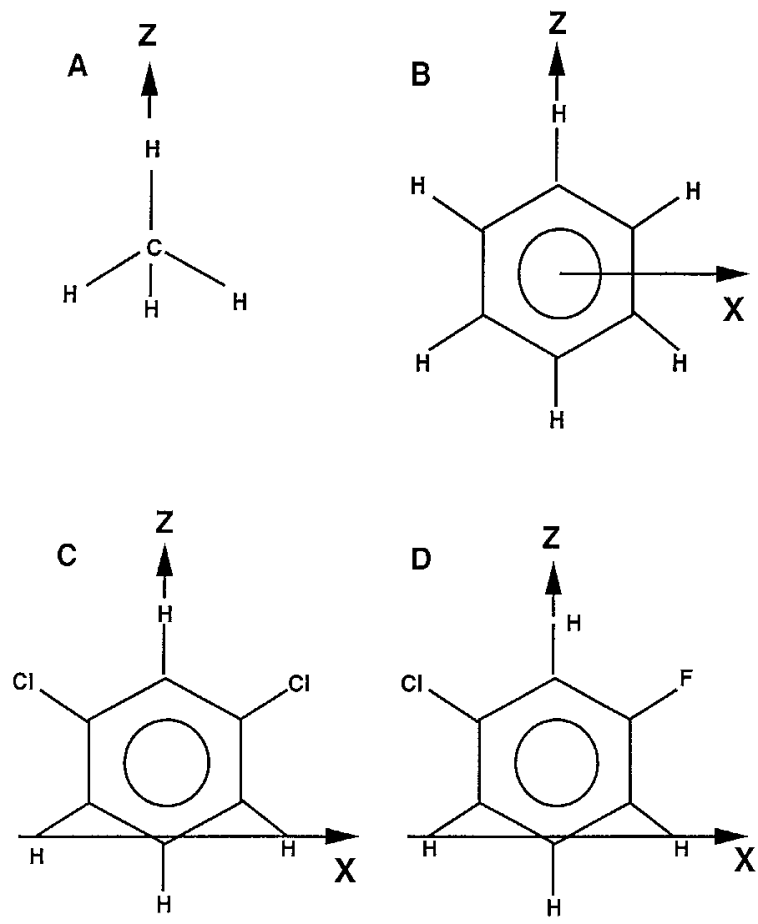

Figure 5 Structure of molecules requiring different numbers of elements of ordering matrix to describe the orientation along with the coordinate system to be chosen: (A) methane, (B) benzene, (C) $m$-dichlorobenzene, and (D) $m$-fluoroiodobenzene. 
tral splittings in the tetrahedral molecules have been discussed in the section on the vibrational corrections to the molecular geometry.

Molecules possessing a point group symmetry $D_{2 d}, C_{3}$, and a higher axis of symmetry require only one independent element of the ordering matrix to describe their orientation. In the example chosen in Fig. 5(B), for a molecule with $C_{3 v}$ symmetry, the $Z$ axis is taken to be along the threefold axes of symmetry. Element $S_{Z Z}$ of the ordering matrix therefore describes the orientation of such molecules.

For molecules possessing $C_{2 v}, D_{2}$, and $D_{2 h}$ point group symmetry, the number of independent elements required to describe the orientation reduces to two. Figure 5(C) shows a molecule with $C_{2 v}$ symmetry, and for the chosen coordinate system, $S_{X X}$ and $S_{Y Y}$ are sufficient to describe the orientation.

Figure 5(D) shows an example of the molecule possessing only a plane of symmetry. If the two coordinate axes are chosen to be in the plane of the symmetry, three independent elements of the ordering matrix are needed to describe its orientation. Elements $S_{X X}, S_{Y Y}$, and $S_{X Y}$ of the matrix completely specify its molecular orientation.

For molecules without a plane of symmetry, all five elements of the ordering matrix are essential to describe their orientation.

\section{Order Parameter and Experimental Conditions}

The ordering of the molecule in the nematic phase depends on the temperature and solute concentration. As the concentration of the solute is increased, the ordering generally decreases unless there are solute-solvent interactions. Similarly, ordering generally increases with a decrease in temperature. It is interesting to point out that in a study on the molecule methanol oriented in the liquid crystal 4- $n$-octyloxybenzoic acid, unusual orientational behavior has been observed (18). The order parameter has been measured as a function of temperature and at two different concentrations. There is no steady increase of the order parameter with a decrease in temperature, but the plot of the order parameter as a function of temperature goes through a maximum. This unusual behavior of methanol is interpreted as due to association. Any change in the order parameter results in a change in the strength of the dipolar interaction. Hence, the spectra of the given molecule in a particular liquid crystal are not the same when recorded at different temperatures or concentrations.

\section{MATERIALS AND METHODS}

\section{Preparation of the Solution}

Solutions are prepared by mixing appropriate amounts of solute and solvent. Solute concentration is maintained such that the liquid crystallinity is not destroyed and it is usually 7-8 wt $\%$. The solutions are homogenized by repeated heating of the mixtures to the isotropic phase and then cooling back to the nematic phase, with physical shaking or vortex mixing. It should be noted that inhomogeneities as a result of temperature or concentration gradient within the sample provide spectra with differential line broadening. This differential line broadening is in addition to inhomogeneity of the external magnetic field. In normal superconducting magnets, inhomogeneity due to the external static magnetic field can be reduced by spinning the sample about the vertical axis without destroying the orientation. Typical line widths around $1-2 \mathrm{~Hz}$ can be obtained for the proton spectra, depending upon the choice of the liquid crystal, experimental conditions, and preparation of the sample. It is appropriate to mention that when the nematic liquid crystal ZLI-1167 (an eutectic mixture of propyl, pentyl, and heptyl bicyclohexyl carbonitrile) [Fig. 3(D)] is used, sharp lines are generally observed.

\section{Identification of the Spin System}

As mentioned earlier, the dominant parameters in the NMR spectra of oriented molecules are usually intramolecular dipolar couplings. As far as the nomenclature of the NMR spectra is concerned, it is identical to that followed in conventional high-resolution NMR studies in liquids. However, the definition of weak and strong coupling in the spectra of oriented molecules depends on the strength of both the dipolar couplings $\left[D_{i j}\right.$ (dir)'s] and the indirect spin-spin couplings compared to chemical shifts, unlike the strength of only the indirect spin-spin couplings $\left(J_{i j}\right.$ 's) in the conventional liquid-state NMR spectra. The strength of the dipolar couplings may usually vary from a few hertz to a few kilohertz. The spectra may therefore be very complex, with a spread of several kilohertz, and are generally strongly coupled, particularly in the thermotropic 
nematic liquid crystals normally used unless heteronuclei are involved $(19,20)$. The second important difference is that a group which is magnetically equivalent and provides a single resonance line in the isotropic phase gives rise to a multiplicity of lines in oriented systems. For example, in benzene in the isotropic phase, all six protons have the same chemical shift, and also the indirect spin-spin couplings do not affect the spectrum, giving rise to a single resonance line compared to a complex spectrum in the oriented phase. The spectrum of benzene is designated as $\mathrm{AA}^{\prime} \mathrm{A}^{\prime \prime} \mathrm{A}^{\prime \prime \prime} \mathrm{A}^{\prime \prime \prime \prime} \mathrm{A}^{\prime \prime \prime \prime \prime}$. Furthermore, if all nuclei within a magnetically equivalent group are equally coupled to one another, the group is called "fully equivalent." Such a group is denoted as $A_{n}$, where $n$ is the number of fully equivalent spin- $\frac{1}{2}$ nuclei. For oriented molecules, even fully equivalent groups such as $A_{n}$ give rise to a multiplicity of " $n$ " transitions with a separation equal to $3 D_{i j}$ (dir) between adjacent transitions. The intensity distribution of the transitions corresponds to coefficients of the binomial expansion. For example, methyl protons of $\mathrm{CH}_{3} \mathrm{CN}$, a fully equivalent group, give rise to a singlet in the isotropic phase because indirect spin couplings between the equivalent protons of the methyl group do not influence the spectrum. In the anisotropic phase, the nomenclature of the spin system is $A_{3}$ and the dipolar couplings between the protons of the methyl group, although equal between any two methyl protons, result in a triplet with the intensity ratio of $1: 2: 1$.

The molecules with a "tetrahedral (Td)" or higher symmetry in general should not orient and must give a single resonance line in both the isotropic as well as oriented phases. However, as mentioned earlier, a multiplicity of spectral lines are observed in such systems in the oriented phase.

\section{Analyses of the Spectra}

For $N$ interacting spin- $\frac{1}{2}$ nuclei, the number of allowed transitions is given by ${ }^{2 N} C_{N-1}$. For example, for systems with five, six, seven, and eight interacting spins, there are 210, 792, 3003, and 11,440 allowed transitions, respectively. Hence, for every addition of an interacting spin, the number of allowed transitions increases by nearly fourfold. Beyond eight interacting spins, the spectra usually become too complicated and the anal- ysis will be tedious. However, in the literature there are several examples where the spectra of nine and 10 interacting spins have been analyzed (21-24). The first-order analyses of the spectra similar to those of liquids are generally not possible, and one has to diagonalize the Hamiltonian numerically, adapting least-square-fit techniques using computers. Several iterative computer programs suitably modified by the inclusion of the dipolar coupling parameters are commonly used.

\section{Analyses of the Spectra Using a Computer Program}

The programs normally employed are named LAOCOONOR (25) and an NMR spectrometer manufacturing Bruker version of the program PANIC. The starting parameters for the iterative analyses of the spectra are the chemical shifts, indirect spin-spin couplings, and direct dipolar couplings. The dipolar couplings and chemical shift parameters are unknown for an oriented system. However, starting parameters for the chemical shifts and indirect spin-spin couplings are usually taken as values in the isotropic media. The dipolar couplings are calculated assuming the molecular geometry and certain guessed values for the orientational tensor components. Using these dipolar couplings, a spectrum is simulated. When there is visual resemblance of the simulated spectrum with the experimental one, certain specific experimental lines are assigned to theoretical ones and the parameters are varied iteratively. The iteration procedure is continued until all experimental lines are assigned to the simulated ones and the root-mean-square error between the calculated and experimental line positions reaches a global minimum. The strategy adapted for the analysis of the spectra is shown in the form of a flowchart in Fig. 6. A program called XSIM (26) works on SUN, SGI, and RS6000 computers.

\section{Alternate Strategy for Analyses of the Spectra}

In the iterative strategy discussed above, the analysis can at times be tedious if the spectra are very complex. Basic ingenuity lies in the judicious choice of the starting parameters. The trial spectrum computed should closely resemble the experimental spectrum such that a sufficient num- 


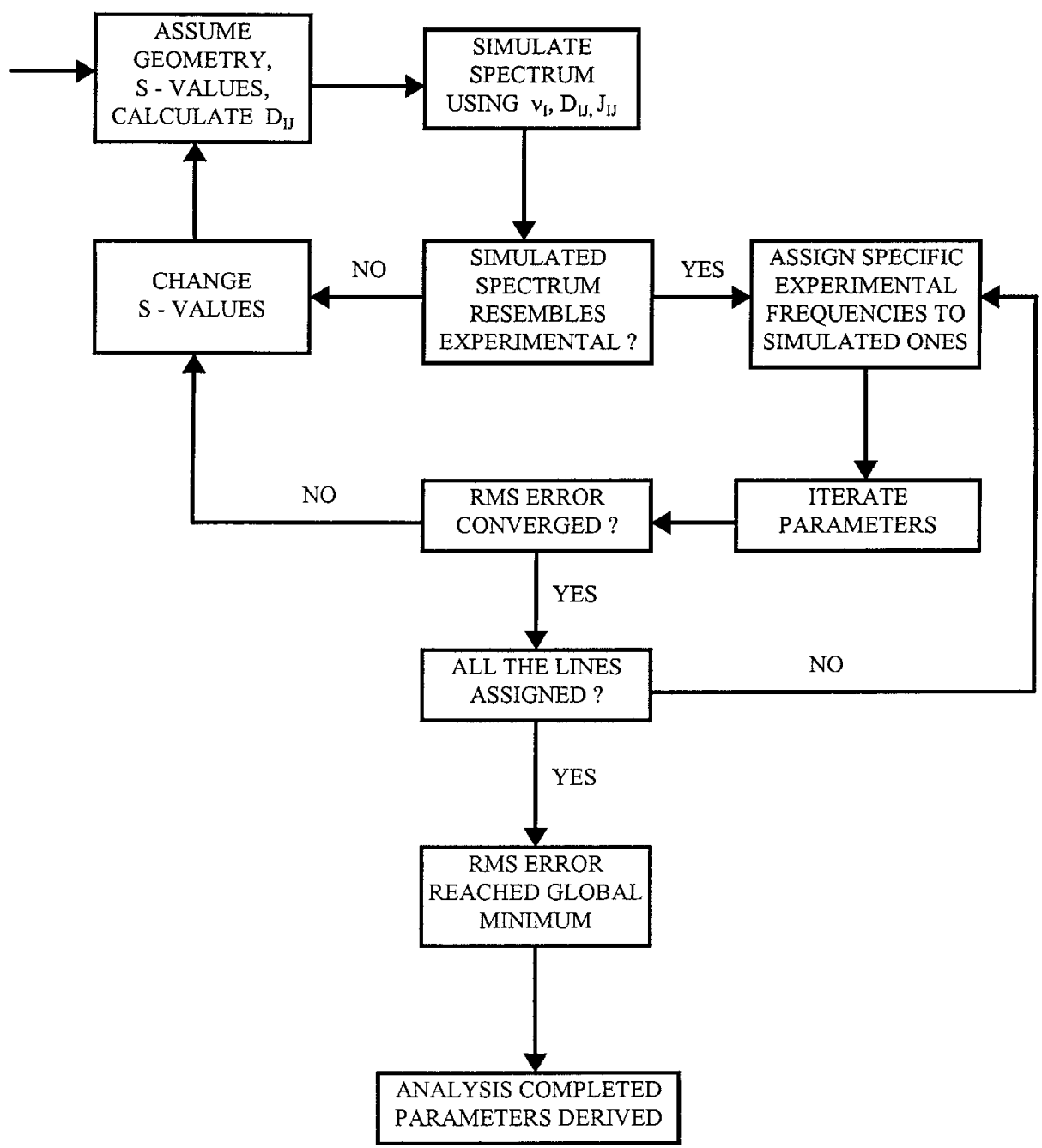

Figure 6 Flowchart showing the strategy adopted for analyses of the NMR spectra of oriented molecules.

ber of transitions can be assigned for subsequent iteration and refinement of the parameters. It is the intuition, experience, and, at times, also the luck of workers in the field that the trial spectra can be simulated with reasonable resemblance to the experimental one so that the complicated spectra can be analyzed. To overcome the tedium of such analyses, a program called DANSOM (27, 28) has been developed for automatic analysis using an alternative strategy which does not require line assignments. These methods always make use of the full information content of an NMR spectrum, i.e., the total line shape. This information is supplied to the computer as the $n$-dimensional vector $\mathbf{S}$ of digitized signal intensities $\mathbf{S}_{i}$ which are ordered according to increasing or decreasing discrete frequencies $\omega_{i}$. The basic algorithm relies on a matrix method derivable from a generalized formulation of the leastsquares problem. Details of the theoretical basis of this automatic analysis are available in the literature (29) and are too voluminous to discuss in this article. However, it is important to note that these programs circumvent the tedium of line assignments, and in many cases are found to yield satisfactory results. The programs dedicated to the NMR instrument, called WIN-DAISY (30) and PERCH (31), are now available and work on PC's equipped with Windows NT and Windows 95, respectively. The programs call the experimental spectra directly onto the PC from the spectrometer, and automatic analysis is carried out by varying over a wide range, iteratively, all parameters defining the spectrum. 


\section{Comment on the Heteronuclear Indirect Spin - Spin Couplings}

In the Hamiltonian given in Eq. [1], the first and the second terms contribute to the diagonal elements, and the third term to the off-diagonal elements of the matrix. The chemical shift separation between the heteronuclei is of the order of megahertz, whereas the dipolar and indirect spin-spin couplings are several orders of magnitude smaller. Consequently, the contributions of off-diagonal elements to the transition frequencies are negligible. Therefore, such spectra are essentially influenced by $J_{i j}+2 D_{i j}$ (dir). Hence, the NMR spectra of oriented molecules do not permit the separate determination of $J_{i j}$ and $D_{i j}$ (dir) between heteronuclei. Analysis of the spectrum is, however, not difficult, because whatever the value is of heteronuclear $J_{i j}$, correspondingly, $D_{i j}$ (dir)'s get altered and the spectrum remain's invariant. Furthermore, for the determination of heteronuclear structural information, precise values of dipolar couplings are essential. Normally, the indirect spin-spin couplings determined from the spectra in the isotropic media are used to derive direct dipolar couplings. However, for the precise determination of heteronuclear $J_{i j}$ in the anisotropic medium itself, the technique of mixed liquid crystals of opposite diamagnetic anisotropies $(32,33)$ is used, and the methodology is discussed below.

\section{USE OF MIXED LIQUID CRYSTALS OF OPPOSITE DIAMAGNETIC SUSCEPTIBILITY ANISOTROPIES}

Depending upon the sign of the diamagnetic susceptibility anisotropy of the liquid crystal, the preferential orientation of the director is along or orthogonal to the magnetic field, and the sign of the $S$ value can be either positive or negative, respectively. From Eq. [10], it can be seen that dipolar couplings will have opposite signs depending on the sign of the $S$ value. It has been observed $(32,33)$ that the dipolar couplings in a mixture of liquid crystals of opposite diamagnetic susceptibilities anisotropies vary gradually with the relative concentration of the solvents. However, near critical concentration, there is an abrupt change in the value of the dipolar couplings to half or twice, with opposite signs depending on the direction of approach of the critical point. That means the orientation of the director changes from either parallel to perpendicular orientation, or vice versa. A close examination of the results in the vicinity of the critical point reveals the coexistence of two spectra corresponding to both orientations of the director of the liquid crystal along and orthogonal to the magnetic field. A variation of the temperature near the critical concentration also may result in the coexistence of two spectra.

At the critical point, where the spectra are coexisting, the dipolar couplings due to one orientation are not independent of the other and are related by a factor of two or half with opposite signs. When the heteronuclei are involved, although the spectrum in each orientation is influenced by the sum of $J_{i j}+2 D_{i j}$ (dir), the fact that $\left[D_{i j}(\mathrm{dir})\right]_{\|}=-2\left[D_{i j}(\mathrm{dir})\right]_{\perp}$, where $\left[D_{i j}(\mathrm{dir})\right]_{\|}$ and $\left[D_{i j}(\mathrm{dir})\right]_{\perp}$ are the dipolar couplings corresponding to parallel and perpendicular orientations of the director, results in two simultaneous equations, $J_{i j}+2 D_{i j}$ (dir) and $J_{i j}-D_{i j}(\mathrm{dir})$, from which $J_{i j}$ and $D_{i j}$ (dir) can be separately determined.

\section{INTERPRETATION OF DIPOLAR COUPLINGS}

The dipolar couplings derived from the analyses of NMR spectra are subsequently to be interpreted in terms of molecular structure and orientation. Therefore, it implies that the measurable dipolar couplings should be at least equal to the total number of $S$ values and structural parameters to be determined. For example, for a system of $N$ interacting spins and without any symmetry, we have $N(N-1) / 2$ dipolar couplings to determine five elements of the ordering matrix and $3(N-2)$ coordinates. The minimum number of interacting spins required to derive information for such systems is given by the following expression:

$$
[N(N-1) / 2]-5-3(N-2) \geq 0
$$

The minimum value of $N$ required to satisfy the condition of Eq. [15] is 7. For planar systems, the expression gets simplified as

$$
[N(N-1) / 2]-3-(N-2) 2 \geq 0
$$

in which case $N$ should be minimum of 5. Similarly, for planar systems with $C_{2 v}$ symmetry, it can 
be shown that minimum number of interacting spins required should be 4 .

If the number of independent dipolar couplings determined experimentally is less than the total number of the structural and order parameters to be determined, the system is said to be underdetermined, and it is not possible to extract the complete structural information without making some assumptions.

\section{RELATION BETWEEN THE DIPOLAR COUPLINGS AND MOLECULAR STRUCTURE}

Arithmetic expressions to relate dipolar couplings to the structural and orientational parameters of the molecules can be written. These expressions can be solved numerically using iterative procedures, or wherever possible analytically, to derive information on the molecular geometry. A few examples are discussed below.

\section{A Linear Molecule with Three Interacting Spins}

This is the simplest molecule whose structure can be studied using this technique. For example, if we take 1,2 , and 3 to be the three protons, then there are three dipolar couplings. Using Eq. [10], they can be written as

$$
\begin{aligned}
& D_{12}(\text { dir })=-K_{12} \cdot S_{12} / r_{12}^{3} \\
& D_{13}(\text { dir })=-K_{13} \cdot S_{13} / r_{13}^{3} \\
& D_{23}(\text { dir })=-K_{23} \cdot S_{23} / r_{23}^{3}
\end{aligned}
$$

With the molecule being linear, $S_{12}=S_{13}=S_{23}$. Taking the ratio

$$
\left[D_{12}(\operatorname{dir}) / D_{13}(\operatorname{dir})\right]=\left(r_{13} / r_{12}\right)^{3}
$$

we can thus determine the relative internuclear distances from the two dipolar couplings. The order parameter can be determined from any of the three dipolar couplings if one of the internuclear distances is known.

\section{Four Spin Systems with $C_{3 v}$ Symmetry}

An example of such a system is a rotating methyl group with another atom situated on the symmetry axis, as shown in Fig. 7. There are two dipolar

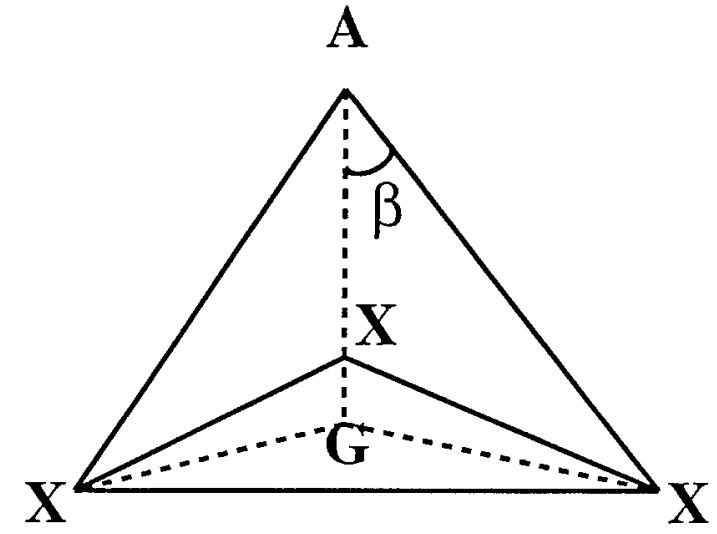

Figure 7 Schematic showing a four-spin system of the type $A X_{3}$ with $C_{3 v}$ symmetry.

couplings that provide the desired information as follows. If the dipolar coupling within the methyl protons and the dipolar couplings between the methyl proton and the other atom are denoted as $D_{X X}$ and $D_{A X}$, respectively, then

$$
D_{A X}(\text { dir })=\frac{-\left(\gamma_{A} \gamma_{X} h\right)}{4 \pi^{2}} \frac{1}{r_{A X}^{3}} S_{A X}
$$

If $\beta$ is the angle the $A X$ axis makes with the $C_{3}$-symmetry axis, and defining $S_{C_{3}}$ as the order parameter along the $C_{3}$ axis of symmetry, $S_{A X}$ can be expressed in terms of $S_{C_{3}}$ as

$$
\begin{aligned}
S_{A X}= & S_{C_{3}} \cos ^{2} \beta-(1 / 2) S_{C_{3}} \sin ^{2} \beta \\
& -\left(S_{C_{3}} / 2\right) \cos ^{2} 90
\end{aligned}
$$

As the last term of Eq. [22] is zero,

$$
S_{A X}=S_{C_{3}} \cos ^{2} \beta-(1 / 2) S_{C_{3}} \sin ^{2} \beta
$$

expressing $\sin ^{2} \beta$ of Eq. [23] in terms of $\cos ^{2} \beta$

$$
S_{A X}=S_{C_{3}} \cos ^{2} \beta-S_{C_{3}} / 2\left(1-\cos ^{2} \beta\right)
$$

Rearranging of Eq. [24] results in

$$
S_{A X}=S_{C_{3}} / 2\left(3 \cos ^{2} \beta-1\right)
$$

Using Eq. [25] for $S_{A X}$ in Eq. [21]

$$
D_{A X}(\text { dir })=\frac{-\left(\gamma_{A} \gamma_{X} h\right)}{4 \pi^{2}} \frac{1}{r_{A X}^{3}}\left[S_{C_{3}}\left(3 \cos ^{2} \beta-1\right) / 2\right]
$$

The dipolar coupling $D_{X X}(\mathrm{dir})$ is given by

$$
D_{X X}(\text { dir })=\frac{-h^{2}\left(\gamma_{X}^{2}\right)}{4 \pi^{2} r_{X X}^{3}}\left(-S_{C_{3}} / 2\right)
$$


The ratio

$$
\begin{aligned}
{\left[D_{A X}(\mathrm{dir}) / D_{X X}(\mathrm{dir})\right]=} & -\left(\gamma_{A} / \gamma_{X}\right)\left(r_{X X} / r_{A X}\right)^{3} \\
& \cdot\left(3 \cos ^{2} \beta-1\right) / 2 \quad[28]
\end{aligned}
$$

If $\alpha$ is the $\mathrm{HCH}$ bond angle, from basic trigonometry it can be shown that

$$
\sqrt{ } 3 \sin \beta=r_{X X} / r_{A X}=2 \sin (\alpha / 2)
$$

Equation [29] can be rewritten as

$$
\left(r_{X X} / r_{A X}\right)^{2}=3 \sin ^{2} \beta=3\left(1-\cos ^{2} \beta\right)
$$

The simple arithmetic manipulation of Eq. [30] gives

$$
-\left(r_{X X} / r_{A X}\right)^{2}+2=3 \cos ^{2} \beta-1
$$

Substituting Eq. [31] into Eq. [28] for the ratio of dipolar couplings, we get

$$
\begin{aligned}
{\left[D_{A X}(\operatorname{dir}) / D_{X X}(\operatorname{dir})\right]=} & \left(\gamma_{A} / \gamma_{X}\right)\left(r_{X X} / r_{A X}\right)^{3} \\
& \cdot\left[\left(r_{X X} / r_{A X}\right)^{2}-2\right]
\end{aligned}
$$

Equation [32] can be used to derive information on the relative internuclear distance.

\section{Four Spin Systems with $C_{2 v}$ Symmetry}

The four spin systems with $C_{2 v}$ symmetry shown in Fig. 8 have four independent dipolar couplings. Two $S$ values are required to define the molecular orientation. The dipolar couplings for different spin pairs are referred to as $D_{1}$ (dir), $D_{2}$ (dir), $D_{3}$ (dir), and $D_{4}$ (dir), and the corresponding internuclear distances are denoted as $r_{1}, r_{2}, r_{3}$, and $r_{4}$, respectively. For brevity, these dipolar couplings are referred to as $D_{1}, D_{2}, D_{3}$, and $D_{4}$, respectively in this section. Using Eq. [10], representing constant $K_{i j}$ as $K$ for simplicity, the different dipolar couplings are written as

$$
\begin{gathered}
D_{1}=\left(-K / r_{1}^{3}\right) S_{X X} \\
D_{2}=\left(-K / r_{2}^{3}\right) S_{X X} \\
D_{3}=\left(-K / r_{3}^{3}\right)\left[S_{X X} \cos ^{2} \theta+S_{Y Y} \sin ^{2} \theta\right] \\
D_{4}=\left(-K / r_{4}^{3}\right)\left[S_{X X} \cos ^{2} \varphi+S_{Y Y} \sin ^{2} \varphi\right]
\end{gathered}
$$

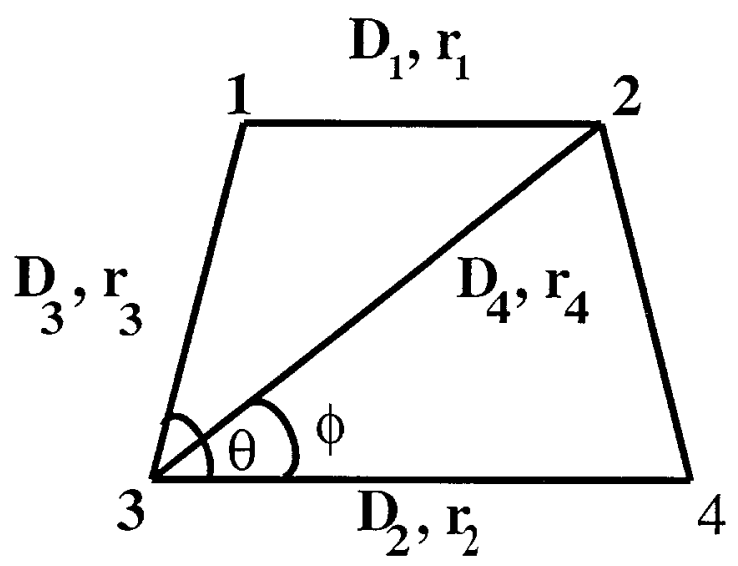

Figure 8 Schematic showing the numbering of spins and representation of different dipolar couplings, and the corresponding distances of a four-spin system with $C_{2 v}$ symmetry.

The distance ratio $r_{2} / r_{1}$ is then given by

$$
\left(r_{2} / r_{1}\right)=\left(D_{1} / D_{2}\right)^{1 / 3}
$$

From knowledge of trigonometry, we can write

$$
\begin{gathered}
\cos \theta=\left(r_{2}-r_{1}\right) / 2 r_{3} \\
\cos \varphi=\left(r_{2}+r_{1}\right) / 2 r_{4} \\
r_{4}^{2}=r_{2}^{2}+r_{3}^{2}-2 r_{2} r_{3} \cos \theta
\end{gathered}
$$

Substituting for $\cos \theta$ in Eq. [40] gives

$$
r_{4}^{2}=r_{3}^{2}+r_{2} r_{1}
$$

Using Eq. [38] in Eq. [35] and expressing $\sin ^{2} \theta$ as $1-\cos ^{2} \theta$,

$$
\begin{aligned}
D_{3}= & -\left(K / r_{3}^{3}\right)\left[S_{X X}\left(r_{2}-r_{1}\right)^{2} / 4 r_{3}^{2}\right. \\
& \left.+S_{Y Y}\left(1-\left(r_{2}-r_{1}\right)^{2} / 4 r_{3}^{2}\right)\right]
\end{aligned}
$$

Using $S_{X X}$ obtained from Eq. [33] and rearranging Eq. [42]

$$
\begin{aligned}
D_{3} r_{3}^{3}= & r_{1}^{3} D_{1}\left(r_{2}-r_{1}\right)^{2} / 4 r_{3}^{2} \\
& -K S_{Y Y}\left(4 r_{3}^{2}-\left(r_{2}-r_{1}\right)^{2} / 4\right)
\end{aligned}
$$


Multiplying Eq. [431 by $r_{3}^{2}$

$$
\begin{aligned}
D_{3} r_{3}^{5}= & -r_{1}^{3} D_{1}\left(\left(r_{2}-r_{1}\right) / 2\right)^{2} \\
& +K S_{Y Y}\left[\left(4 r_{3}^{2}-\left(r_{2}-r_{1}\right)^{2}\right) / 4\right]
\end{aligned}
$$

Rearranging Eq. [44]

$$
\begin{aligned}
K S_{Y Y}= & {\left[-D_{3} r_{3}^{5}+r_{1}^{3} D_{1}\left(\left(r_{2}-r_{1}\right) / 2\right)^{2}\right] / } \\
& {\left[\left(4 r_{3}^{2}-\left(r_{2}-r_{1}\right)^{2}\right) / 4\right] }
\end{aligned}
$$

A similar expression for $K S_{Y Y}$ can be obtained in terms $D_{4} r_{4}$ using Eq. [36]

$$
\begin{aligned}
K S_{Y Y}= & -\left[D_{4} r_{4}^{5}+r_{1}^{3} D_{1}\left(\left(r_{2}+r_{1}\right) / 2\right)^{2}\right] / \\
& {\left[\left(4 r_{4}^{2}-\left(r_{2}+r_{1}\right)^{2}\right) / 4\right] }
\end{aligned}
$$

Since the left-hand side of Eqs. [45] and [46] are equal, the right-hand side must be equal. Furthermore, the denominator of the right-hand side of the Eq. [46] can be simplified as

$$
\begin{aligned}
& =1 / 4\left[\left(4 r_{4}^{2}-\left(r_{2}+r_{1}\right)^{2}\right) / 4\right] \\
& =1 / 4\left[4 r_{4}^{2}-r_{2}^{2}-r_{1}^{2}+2 r_{2} r_{1}\right]
\end{aligned}
$$

Using Eq. [41]

$$
=1 / 4\left[4 r_{3}^{2}+4 r_{2} r_{1}-r_{2}^{2}-r_{1}^{2}+2 r_{2} r_{1}\right]
$$

Equation [49] is simplified as

$$
=1 / 4\left[4 r_{3}^{2}-\left(r_{2}-r_{1}\right)^{2} / 4\right]
$$

This is identical to the denominator of the righthand side of Eq. [45]. Hence, the numerators of the right-hand side of Eqs. [45] and [46] must be equal.

$$
\begin{aligned}
& -D_{3} r_{3}^{5}+r_{1}^{3} D_{1}\left(\left(r_{2}-r_{1}\right) / 2\right)^{2} \\
& \quad=-D_{4} r_{4}^{5}+r_{1}^{3} D_{1}\left(\left(r_{2}+r_{1}\right) / 2\right)^{2}
\end{aligned}
$$

Dividing Eq. [51] by $r_{1}^{5}$

$$
\begin{aligned}
& -D_{3}\left(r_{3} / r_{1}\right)^{5}+D_{1}\left(\left(r_{2}-r_{1}\right) / 2 r_{1}\right)^{2} \\
& =-D_{4}\left(r_{4} / r_{1}\right)^{5}+D_{1}\left(\left(r_{2}+r_{1}\right) / 2 r_{1}\right)^{2}
\end{aligned}
$$

On rearranging Eq. [52], we get

$$
\begin{aligned}
& -D_{3}\left(r_{3} / r_{1}\right)^{5}+D_{4}\left(r_{3} / r_{1}\right)^{5} \\
& \quad=D_{1}\left[\left(\left(r_{2}+r_{1}\right)^{2} / 4 r_{1}^{2}\right)-\left(\left(r_{2}-r_{1}\right)^{2} / 4 r_{1}^{2}\right)\right]
\end{aligned}
$$

The right-hand side of Eq. [53] can be simplified as follows:

$$
\begin{gathered}
=D_{1} / 4 r_{1}^{2}\left[\left(r_{2}+r_{1}\right)^{2}-\left(r_{2}-r_{1}\right)^{2}\right] \\
=D_{1}\left[r_{2} / r_{1}\right]
\end{gathered}
$$

Using Eq. [37], Eq. [55] can be written as

$$
=D_{1}\left[D_{1} / D_{2}\right]^{1 / 3}
$$

Replacing the right-hand side of Eq. [53] by Eq. [56], we get

$$
-D_{3}\left(r_{3} / r_{1}\right)^{5}+D_{4}\left(r_{4} / r_{1}\right)^{5}-D_{1}\left(D_{1} / D_{2}\right)^{1 / 3}=0
$$

Using Eq. [41] for $r_{4}$, the second term of Eq. [57] can be expressed as

$$
\begin{gathered}
=D_{4}\left[\left(r_{3}^{2}+r_{2} r_{1}\right) / r_{1}\right]^{5} \\
=D_{4}\left[\left(r_{3} / r_{1}\right)^{2}+\left(r_{2} / r_{1}\right)\right]^{5 / 2}
\end{gathered}
$$

The ratio of $r_{2} / r_{1}$ can be expressed in terms of the ratios of $D_{1} / D_{2}$ using Eq. [37]

$$
=D_{4}\left[\left(r_{3} / r_{1}\right)^{2}+\left(D_{1} / D_{2}\right)\right]^{5 / 2}
$$

Thus, Eq. [60] can be written in terms of the ratios of dipolar couplings and distances as follows:

$$
\begin{gathered}
-D_{3}\left(r_{3} / r_{1}\right)^{5}+D_{4}\left[\left(r_{3} / r_{1}\right)^{5}+\left(D_{1} / D_{2}\right)^{1 / 3}\right]^{5 / 2} \\
-D_{1}\left(D_{1} / D_{2}\right)^{1 / 3}=0
\end{gathered}
$$

Furthermore, if the four protons are at the corners of a planar rectangle, then $r_{1}=r_{2}$ and $D_{1}=$ $D_{2}$, and Eq. [61] gets simplified as

$$
-D_{3}\left(r_{3} / r_{1}\right)^{5}+D_{4}\left[\left(r_{3} / r_{1}\right)^{5}+1\right]^{5 / 2}-D_{1}=0
$$

Thus, using the four experimentally determined dipolar couplings, and using Eqs. [61] and [62], it is possible to derive internuclear distance ratios. 


\section{Computer Program to Determine the Structure}

As can be seen from the above examples, even for a simple case of four interacting spins, the relation between the dipolar couplings and the molecular geometry are governed by the expressions of the fifth order. Thus, the analytical solutions to these expressions are no longer easy and must be found iteratively by computer. For this, the computer program SHAPE (34) is available. This program, from experimental dipolar couplings and a trial set of order parameters and geometrical parameters, iteratively determines the shape of the nuclear skeleton and the elements of the ordering matrix by a least-square-fit technique. All independent dipolar couplings are fitted in the program by minimizing the function

$$
\sum\left(D_{i}(\operatorname{expt})-D_{i}(\text { calcd })\right) / \sigma_{1}^{2}
$$

where $i$ refers to different dipolar couplings and $\sigma_{I}$ is the variance of $D_{i}$ (expt). Also, different weights may be given to different couplings in the program to provide a weighted least-square fit of structural and orientational parameters.

\section{VIBRATIONAL CORRECTIONS TO MOLECULAR GEOMETRY}

In general, molecules whose structures are determined are strictly not rigid. Molecules undergo vibrational motions in a period much shorter than the NMR time scale. A structure determined without taking these vibrations into account can give rise to internally inconsistent structures. For example, in a linear three-spin system of the type $A B C$, there is a shrinkage effect (35). That is, the distance $A C$ computed is always shorter than $A B+B C$. Moreover, the mean values determined for the internuclear distances using different techniques such as X-ray diffraction, infrared spectroscopy, microwave spectroscopy, and electron diffraction make use of the time average of different functions of internuclear distances. For example, electron diffraction provides $\langle r\rangle$ and microwave provides $\left\langle r^{-2}\right\rangle$. That is, the vibrational motions are weighted differently in different techniques. Hence, for direct comparison between the structures determined using different techniques, and to get an internally consistent structure, it is necessary to correct the dipolar couplings for molecular vibrations. Equation [10] then has to be averaged over vibrational motions, and can be written as

$$
\left\langle D_{i j}(\text { dir })\right\rangle_{\mathrm{vib}}=-K_{i j}\left\langle S_{i j} r_{i j}^{-3}\right\rangle
$$

In addition to vibrations, the dipolar couplings may be affected owing to anisotropic interactions of the solute with a liquid crystalline environment. Since the interaction between solute and solvent molecules is expected to depend on details of the configurational changes in the dissolved species, the interplay between molecular internal motion and reorientation also becomes important. This becomes evident when the tetrahedral molecules show unexpected splitting when aligned in liquid crystals. Because every symmetric second-order tensorial property of a tetrahedral system is isotropic by symmetry, such a system should not give rise to second-rank orientation. The observed splitting of a tetrahedral molecule such as methane (17) is explained by taking into account the deviation from the tetrahedral symmetry, through the vibration of these molecules, that induces anisotropy into the interaction with the liquid crystal environment. This interaction is between the anisotropic liquid crystal electric field and the vibrationally induced electric polarizability of the solute. That means one needs to consider the interaction that depends on both the orientation (rotational) and vibration of the solute molecules. Such an interaction is termed vibrational-rotation coupling. This coupling induces a correlation between the two modes. The theory which takes into account the vibrational and rotational coupling in the anisotropic media will treat this solvent solute interaction as a tensorial of the second order (17, 36). The dipolar splitting is then shown to arise from both rigid and nonrigid molecule effects. The nonrigid contribution can be derived from the harmonic forcefield and the rigid molecule effect can be derived from cubic anharmonic forcefields. Furthermore, a lot of information is available in the literature (37) which provides much insight into solvent-solute interactions. This also takes into account the long-range contributions, which depend on the electronic property of the solute with the mean field of the solvent, and the short range contributions, which depend on the size and shape of the solute molecule, to the average orientation. Even the importance of the shape of the solvent molecule has also been emphasized in one study (38). To estimate all these effects accurately for a probe molecule, sufficient 
data must be available from other sources. Thus, to date, the application of these corrections to experimentally determined dipolar couplings to wide range of solutes is still limited. Application of a harmonic vibration correction for the probe molecules is, however, relatively easy if the valence forcefields are available. This harmonic vibrational corrections is discussed below.

If the molecular reorientation time is slow compared with the vibrational period, one can safely assume that the two motions (vibration and rotation) are uncorrelated and the molecule does not change in orientation during vibrations, and the average of $D_{i j}$ is

$$
\left\langle D_{i j}(\mathrm{dir})\right\rangle_{\mathrm{vib}}=-K_{i j}\left\langle S_{i j}\right\rangle\left\langle r_{i j}^{-3}\right\rangle
$$

The quantity $\left\langle r_{i j}^{-3}\right\rangle$ can be evaluated from the dipolar couplings. The ultimate object of the structure determination is to determine the equilibrium structure $\left(r_{e}\right)$. This equilibrium structure is independent of the experimental technique used. This can be evaluated by determining the difference between the observed dipolar coupling $D_{i j}$ (expt) and that calculated from the positions of the nuclei in their equilibrium positions $\left(D_{i j}^{e}\right)$. If the $D_{i j}(\operatorname{expt})$ is close to $D_{i j}^{e}$, then the relationship between the two can be expressed by the Taylor series

$$
\begin{aligned}
D_{i j}(\text { expt })= & D_{i j}^{e}+\Delta X_{i j}\left(\delta D_{i j}^{e} / \delta X\right) \\
& +\Delta Y_{i j}\left(\delta D_{i j}^{e} / \delta Y\right) \\
& +\Delta Z_{i j}\left(\delta D_{i j}^{e} / \delta Z\right) \\
& +1 / 2 \Delta X_{i j}^{2}\left(\delta^{2} D_{i j}^{e} / \delta X^{2}\right) \\
& +1 / 2 \Delta Y_{i j}^{2}\left(\delta^{2} D_{i j}^{e} / \delta Y^{2}\right) \\
& +1 / 2 \Delta Z_{i j}^{2}\left(\delta^{2} D_{i j}^{e} / \delta Z^{2}\right)
\end{aligned}
$$

The quantities $\Delta X_{i j}, \Delta Y_{i j}$, and $\Delta Z_{i j}$ are the mean amplitudes, and $\Delta X_{i j}^{2}, \Delta Y_{i j}^{2}$, and $\Delta Z_{i j}^{2}$ are the mean square amplitudes of vibration in the $X, Y$, and $Z$ directions, respectively. Evaluation of mean amplitudes requires a knowledge of the anharmonic terms in the potential function of the vibrating molecule, and such terms are usually unknown except for certain small molecules. The mean square amplitudes are determined only by harmonic terms and can be calculated. Neglecting the anharmonic corrections gives an effective dipolar coupling constant

$$
\begin{aligned}
D_{i j}(\text { expt })= & D_{i j}^{e}-1 / 2 \Delta Z_{i j}^{2}\left(\delta^{2} D_{i j}^{e} / \delta Z^{2}\right) \\
& -1 / 2 \Delta Y_{i j}^{2}\left(\delta^{2} D_{i j}^{e} / \delta Y^{2}\right) \\
& -1 / 2 \Delta Z_{i j}^{2}\left(\delta^{2} D_{i j}^{e} / \delta Z^{2}\right)
\end{aligned}
$$

The molecular structure calculated after the application of the harmonic corrections to the experimental dipolar couplings is referred to as the $r_{\alpha}$ structure. There may be certain differences between the equilibrium structure $\left(r_{e}\right)$ and the $r_{\alpha}$ structure which are due to the anharmonicity of the vibrational potential. However, the $r_{\alpha}$ structure is internally consistent and comparable with other techniques. For such a correction, the computer program VIBR (39) is available. The normal procedure is to start with molecular geometry, and $S$ values are determined from the experimental dipolar couplings using the SHAPE program. Using the program VIBR, the dipolar couplings are corrected for harmonic vibrations. The corrected values are used for a new fit of the structure and $S$ values. The entire procedure is repeated until self-consistency is achieved. It should be pointed out that these corrections are significant for the directly bonded ${ }^{13} \mathrm{C}-\mathrm{H}$ pairs and can be of the order of $3-4 \%$. The prior requirement for the application of these corrections is the availability of valence forcefields for a particular system.

\section{LIMITATIONS OF THE TECHNIQUE}

As discussed earlier, there should be a sufficient number of dipolar couplings to derive structural and orientational parameters. This prescribes the lower limit on the number of spins for which the structural information can be derived. Theoretically, there is no upper limit on the number of interacting spins for which the structure can be derived. However, there are practical limitations to getting well-resolved spectra and the analysis. When the number of interacting spins exceeds 10 , especially when there is no symmetry in the molecule, the spectra are broad and featureless. This prescribes the upper practical limit unless special procedures are followed.

\section{PRACTICAL EXAMPLES}

As practical examples, analyses of the spectra and the determination of the structural information 
are discussed for a few systems. With the lowering of the symmetry of the molecule, for an identical number of spins, the spectra become more complex. This is demonstrated by taking examples of three systems with $D_{6 h}, C_{2 v}$, and planar symmetries requiring one, two, and three elements of the ordering matrix, respectively, for the specification of the molecular orientation. It must be emphasized that though the practical examples taken are of rigid ring systems, the technique is applicable to flexible molecules as well.

\section{Studies on Benzene $(40,41)$}

Spectra of benzene oriented in liquid crystals have been extensively studied (40-44). Consequent to the sixfold symmetry of benzene, one element of the ordering matrix is sufficient to describe its orientation. There are three different proton dipolar couplings, $D_{12}(\mathrm{dir}), D_{13}$ (dir), and $D_{14}$ (dir), corresponding to ortho, meta, and para protons, respectively.

\section{Analysis of the Spectrum}

All six protons of the benzene are magnetically equivalent, and there is only one chemical shift. The spectrum is therefore symmetric about the center and a chemical shift is chosen to be its center. By symmetry consideration, there are 76 transitions allowed. As a first step, if we neglect the indirect spin-spin couplings, then only the dipolar couplings influence the spectrum. The dipolar couplings are computed assuming regular hexagonal geometry of the ring. For a regular hexagon, the distance between the ortho, meta, and para protons, and thereby the dipolar couplings, are related as

$$
D_{12}(\operatorname{dir}):(1 / 3 \sqrt{ } 3) D_{13}(\operatorname{dir}):(1 / 8) D_{14}(\operatorname{dir}) \quad[68]
$$

where the subscripts correspond to protons of benzene which are numbered sequentially. That is, essentially the parameter $D_{12}($ dir) determines the spread of the spectrum. The change in $S$ value results in the linear expansion or contraction of the spectrum. It is therefore possible to make a good guess of the starting values of dipolar couplings. The spectrum thus calculated resembles the experimental spectrum in such a way that the assignment of almost all lines is obtainable by comparing the spectra. Subsequently, analysis is carried out iteratively by varying all parameters affecting the spectrum.

It is interesting to note that the dipolar couplings can be derived analytically in this case (40). In the 50 transitions observed of the 76 permitted, some transitions do not depend on the scalar couplings and can be easily assigned using the symmetry of benzene. The final analysis can be carried out iteratively.

The results provide a ratio of dipolar couplings $D_{12}\left(\right.$ expt): $D_{13}\left(\right.$ expt): $D_{14}($ expt) as 1:0.1929:0.1247. The same ratio for the equilibrium hexagonal position of protons in the benzene ring is 1 : 0.1924:0.1250. Another study on the molecule (41) provided the ratios of $D_{i j}$ (expt)'s as 1:0.1912: 0.1251. The small differences from the regular hexagonal equilibrium positions are attributed to the neglect of vibrational motions of the molecule. Several studies later concentrated on the determination of the $r_{\alpha}$ structure by taking harmonic vibrations into account. The complete $r_{\alpha}$ structure of benzene including the carbon positions has also been derived $(35,45-47)$. To demonstrate the significance of vibrational corrections, the structural parameters of benzene both corrected and uncorrected for molecular vibrations (35) are reproduced in Table 1 . Structural information is also compared with that derived from other techniques in Table 2.

The results demonstrate the significance of vibrational corrections, especially for ratios containing small distances such as, e.g., the direct $\mathrm{C}-\mathrm{H}$ bond, and are of the order of $4 \%$, whereas the corrections for $\mathrm{H}-\mathrm{H}$ distance ratios usually may be neglected.

\section{Table 1 Structural Parameters of Oriented 1- ${ }^{13}$ C-Benzene (19)}

\begin{tabular}{lll}
\hline & \multicolumn{1}{c}{ Case 1 } & \multicolumn{1}{c}{ Case 2 } \\
\hline $\mathrm{r}_{\mathrm{CC}}$ & $1.398 \AA^{*}$ & $1.398 \AA^{*}$ \\
$\mathrm{r}_{\mathrm{CH}}$ & $1.142_{3} \pm 0.000_{5} \AA^{\dagger}$ & $1.101_{2} \pm 0.000_{5} \AA^{\dagger}$ \\
$\mathrm{r}_{\mathrm{CH}} / \mathrm{r}_{\mathrm{CC}}$ & $0.8171 \pm 0.0004^{\dagger}$ & $0.7877 \pm 0.0004^{\dagger}$ \\
$\mathrm{S}_{11}$ & $0.1546 \pm 0.0001$ & \\
$\mathrm{RMS}$ & $0.50 \mathrm{~Hz}$ & $0.38 \mathrm{~Hz}$ \\
\hline
\end{tabular}

Case $1=$ without vibrational corrections; case $2=$ with vibrational corrections ( $r_{x}$ structure).

* Assumed.

${ }^{\dagger}$ Statistical error: 2.5 times standard error from leastsquare fit. 
Table 2 Comparison of Benzene Structural Parameters from Different Methods (19)

\begin{tabular}{llccc}
\hline Parameter & \multicolumn{1}{c}{ NMR } & Electron Diffraction & Electron Diffraction & Raman \\
\hline $\mathrm{CH}$ & $1.142 \pm 0.001 \AA$ & $1.090 \pm 0.02 \AA$ & $1.116 \pm 0.009 \AA$ & $1.084 \pm 0.005 \AA$ \\
$\mathrm{CC}$ & $1.398 \AA^{*}$ & $1.400 \pm 0.005 \AA$ & $1.401 \pm 0.002 \AA$ & $1.397 \pm 0.001 \AA$ \\
$\mathrm{CH} / \mathrm{CC}$ & $0.817 \pm 0.001$ & $0.778 \pm 0.015$ & $0.796 \pm 0.007$ & $0.776 \pm 0.004$ \\
\hline
\end{tabular}

* Assumed.

\section{Structure of Phenylphosphonic Dichloride} (48)

This is a six-spin system with $C_{2 v}$ symmetry; the proton spectrum is complex with several unknown parameters and therefore is discussed in detail. The proton (Fig. 9) and fully coupled ${ }^{13} \mathrm{C}$ spectrum (Fig. 10) of phenylphosphonic dichloride oriented in the liquid crystal ZLI-1167 have been studied to determine the relative positions of protons, phosphorus, and carbon nuclei in the molecule. Indirect $\mathrm{HH}, \mathrm{HP}, \mathrm{HC}$, and $\mathrm{CP}$ couplings have been determined by the analysis of proton and ${ }^{13} \mathrm{C}$ spectrum in the isotropic phase.

\section{Selection of the Starting Parameters and the Analyses of the Spectra}

The structure of the molecule and the numbering of the spins are given in Fig. 9, along with the experimental spectrum (bottom trace). The proton spectrum of the molecule is dominated by $\mathrm{HH}$ and $\mathrm{PH}$ dipolar couplings. The spin system is of the type $A A^{\prime} B B^{\prime} C X$, where $X$ corresponds to phosphorus nucleus. Analysis of the proton spectrum $\left(A A^{\prime} B B^{\prime} C\right.$ part of $\left.A A^{\prime} B B^{\prime} C X\right)$ or the phosphorus spectrum ( $X$ part of $A A^{\prime} B B^{\prime} C X$ ) provides $\mathrm{PH}$ and $\mathrm{HH}$ dipolar couplings. For analysis of the proton spectrum, input parameters are chemical shifts of protons, indirect spin-spin couplings, and dipolar couplings. An approximate arrangement for the proton and phosphorus positions is required to be assumed to estimate the dipolar couplings. A Cartesian coordinate system has been chosen such that the $X$ axis joins the protons numbered 3 and 5 and the $Z$ axis along the $C_{2}$-symmetry axis of the molecule. Taking a $\mathrm{C}-\mathrm{P}$ distance of $1.83 \AA$ (from other studies of similar molecules), and assuming the regular hexagonal
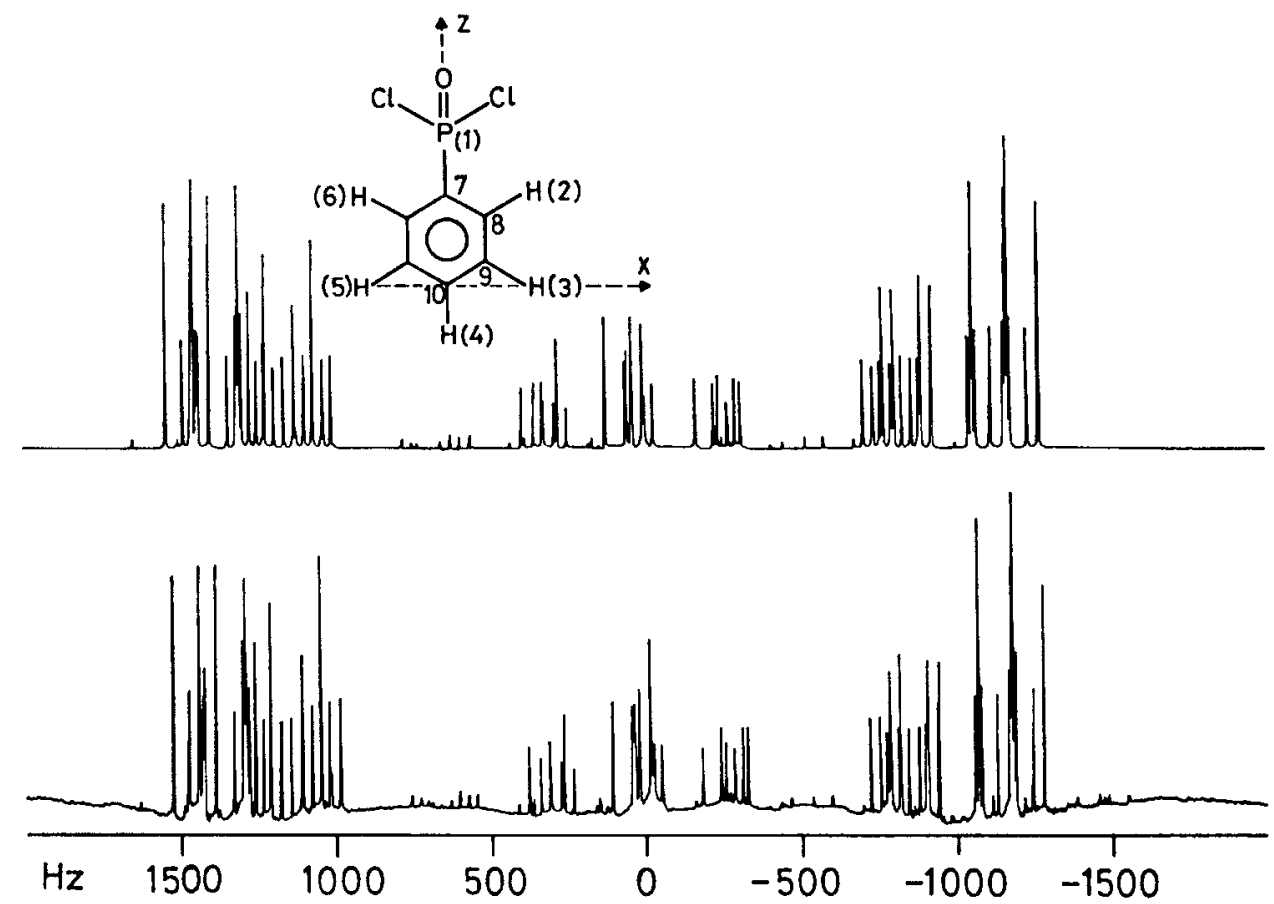

Figure 9 Structure and numbering of the molecule phenylphosphonic dichloride and its proton NMR spectra at $400 \mathrm{MHz}$, oriented in the liquid crystal ZLI-1167. (Bottom trace) Experimental spectrum. (Top trace) Simulated spectrum. The Cartesian coordinate system chosen is also shown. 

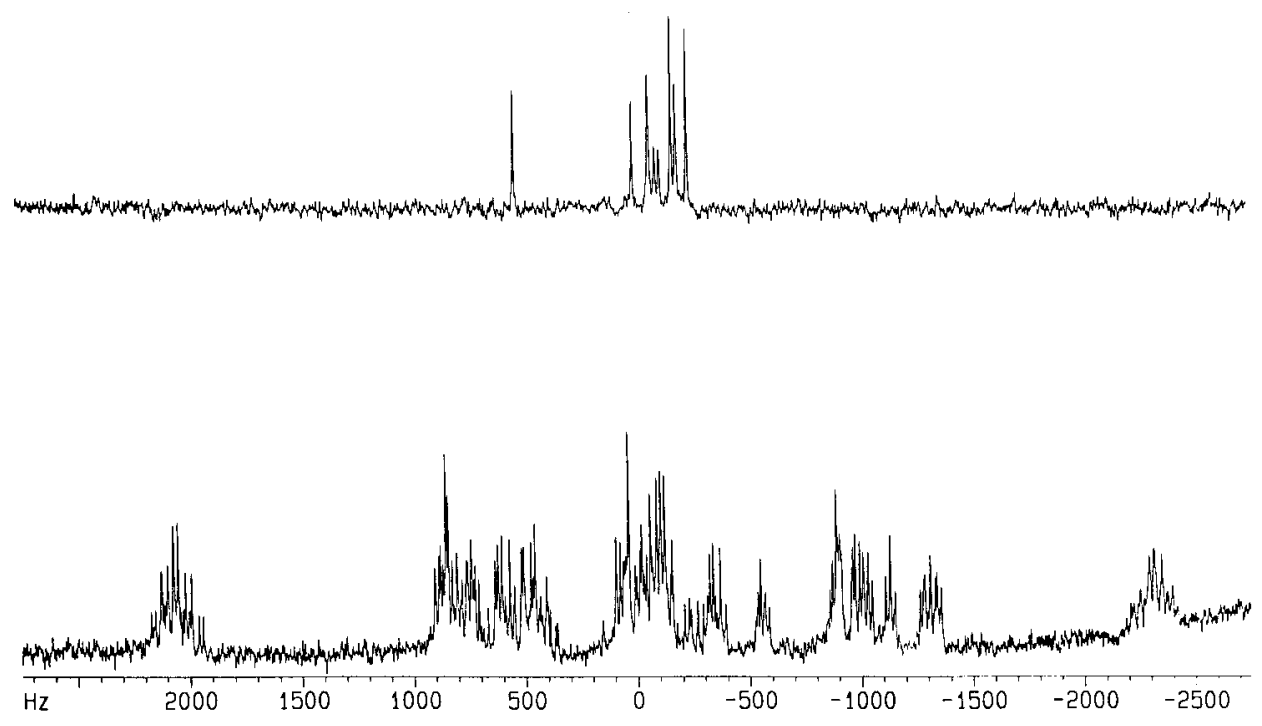

Figure $10{ }^{13} \mathrm{C}$ spectra of phenylphosphonic dichloride oriented in ZLI-1167 at $100 \mathrm{MHz}$. (Bottom trace) Proton coupled. (Top trace) Proton decoupled.

geometry for the phenyl ring, the coordinates of each nuclei were estimated. Two elements of the ordering matrix, $S_{X X}$ and $S_{Z Z}$, required to define the orientation, were guessed and the dipolar couplings calculated. Chemical shifts and indirect spin-spin couplings determined in the isotropic phase and the calculated dipolar couplings were used to simulate the spectrum. The dipolar couplings and chemical shifts have been varied iteratively, keeping the indirect spin-spin couplings constant during the iteration. When the analysis was complete without any ambiguity, there was exact overlap of simulated and experimental spectra. This has been demonstrated by plotting the simulated proton spectrum at the top of the experimental spectrum in Fig. 9.

Knowledge of $\mathrm{CH}$ and $\mathrm{CP}$ dipolar couplings provide information on the carbon positions of the molecule. This information can be obtained from either (a) analysis of the proton or the phosphorus spectrum including ${ }^{13} \mathrm{C}$ satellites, or (b) analysis of the fully coupled ${ }^{13} \mathrm{C}$ spectrum. Analysis of the satellite spectrum is generally imprecise, because all lines are not observable owing to overlap of intense peaks from the abundant protons or phosphorus; the analysis of fully coupled ${ }^{13} \mathrm{C}$ spectrum has therefore been carried out. The molecule has four nonequivalent carbons. Corresponding to each of these nonequivalent carbons, there are four seven-spin systems of the type $A A^{\prime} B B^{\prime} C M X$, where $M$ and $X$ correspond to carbon and phosphorus, respectively. The entire ${ }^{13} \mathrm{C}$ spectrum ( $M$ part of $A A^{\prime} B B^{\prime} C M X$ ) is an overlap of four seven-spin spectra of the type $A A^{\prime} B B^{\prime} C M X$. The analysis of the ${ }^{13} \mathrm{C}$ spectrum corresponding to each of the nonequivalent carbons has to be carried out separately.

Analysis of the proton spectrum provided information on the proton and phosphorus skeleton, and also the values of $S_{X X}$ and $S_{Z Z}$. Using this information, and assuming values of the $\mathrm{C}-\mathrm{C}$ and $\mathrm{C}-\mathrm{H}$ bond distances of 1.395 and $1.1 \AA$, respectively, $\mathrm{CH}, \mathrm{CP}$ dipolar couplings can be estimated and used as the starting parameters for the analysis of the ${ }^{13} \mathrm{C}$ spectra. Indirect spin-spin couplings and the $\mathrm{HH}$ and $\mathrm{PH}$ dipolar couplings determined from the analysis of the proton spectrum were not iterated further. All four simulated spectra when coadded reproduced the experimental ${ }^{13} \mathrm{C}$ spectrum. The individual spectra corresponding to each of the carbons and the coadded spectra are shown in Fig. 11.

\section{Interpretation of the Dipolar Couplings}

The analysis of the proton and the carbon-13 spectra should, in principle, provide 29 independent dipolar couplings. However, for spin systems of the type $A A^{\prime} B B^{\prime} C, A A^{\prime} B B^{\prime} X, A A^{\prime} B B^{\prime} C X$, $A A^{\prime} B B^{\prime} C M X$, etc., when the nuclei $A, A^{\prime}, B, B^{\prime}$ lie nearly at the corners of a rectangle and the orientation of molecule is such that one of the dipolar couplings is very large compared to others (that is, when one of the $S$ values is very large compared to others), it is not possible, in general, to derive all the parameters independently. For example, a situation like this is observed in unsymmetrical paradisubstituted benzenes, espe- 


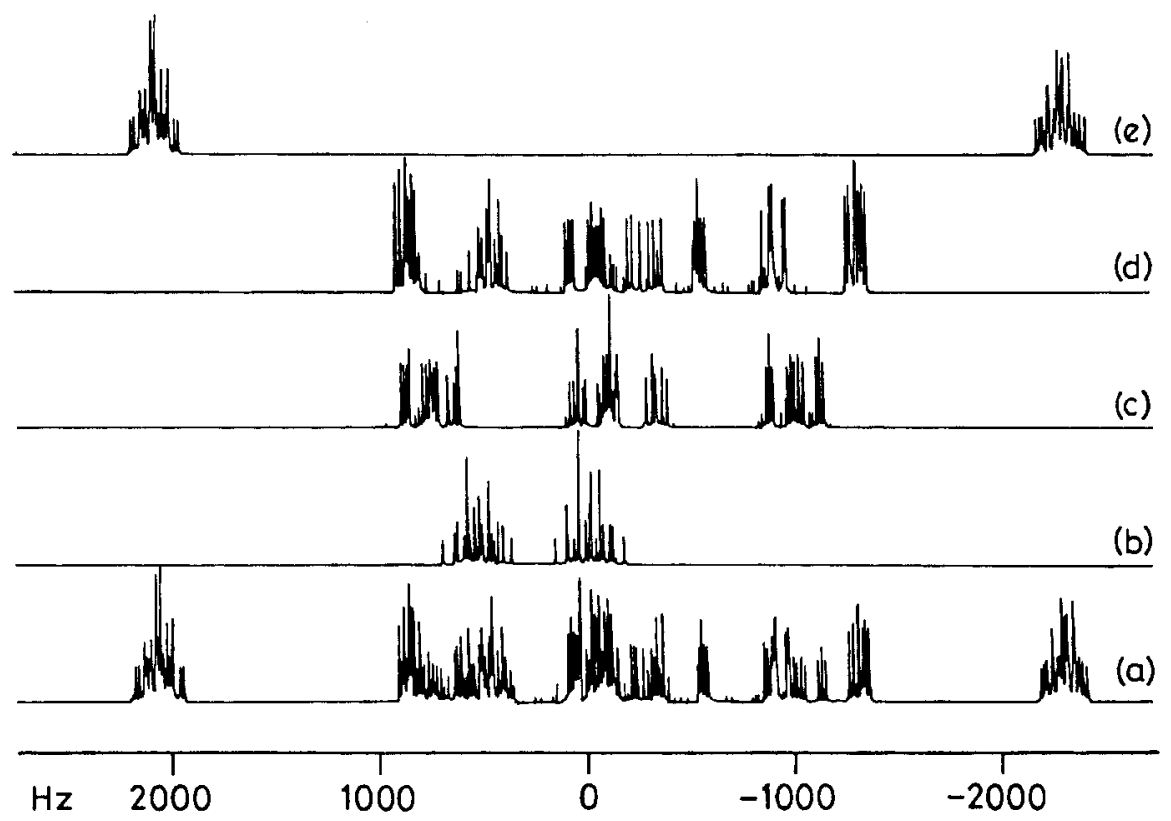

Figure 11 Simulated spectra of various carbons of phenylphosphonic dichloride (b-e) are for the carbons labeled 7,8,9, and 10, respectively; (a) is the superposition of the above four spectra.

cially when they are studied in thermotropic nematic liquid crystals. The variance and covariance matrix of the derived parameters show that the covariances between some of the dipolar couplings and between some of the chemical shifts are large, indicating that these dipolar couplings and chemical shifts cannot be derived independently and precisely from the spectrum. However, careful study of such a variance and covariance matrix reveals that it is possible to choose pairs of dipolar couplings and pairs of chemical shifts whose covariances are very large. These dipolar couplings and chemical shifts when iterated as sums provide precise values. Such spectra are called deceptively simple. Thus, the analyses of such spectra do not provide all possible dipolar couplings independently.

In the present discussion on the molecule phenylphosphonic dichloride, such a situation is observed, and it is not possible to derive all 29 dipolar couplings. Nine dipolar couplings have been determined only as sums of some of the couplings. Thus, only 20 independent dipolar couplings have been determined. Two of these dipolar couplings are needed for determination of the two elements of the ordering matrix. If the distance between the protons numbered 3 and 5 (Fig. 9) is fixed for scaling, then $Z$ coordinates of phosphorus, two carbons and proton along the $C_{2}$ axis and $X$ and $Z$ coordinates of the proton numbered 2 and carbons numbered 8 and 9 are to be determined. Ten independent coordinates fix the complete proton, carbon, and phosphorus positions of the molecule. Thus, the system is overdetermined by eight dipolar couplings. The spectral, structural, and orientational parameters of the molecule are reproduced in Tables 3 and 4 . It should be pointed out that structural parameters are uncorrected for molecular vibrations.

\section{Structure of Benzo(b)tellurophene (49)}

This example clearly depicts the complex nature of the spectrum as the symmetry of the molecule is lowered. The molecule has a planar symmetry with six- and five-membered rings fused together. The six protons forms a spin system of the type $A B C D E F$. The proton spectrum and the numbering of the interacting spins are reproduced in Fig. 12. As can be seen from the figure, the spectrum is very complex compared to previous two examples. The analysis of such a spectrum is very tedious compared to earlier two examples, especially because all chemical shifts in the anisotropic phase and dipolar couplings are unknown for the system. However, the strategy adopted for the 
Table 3 Spectral Parameters of Phenylphosphonic Dichloride Oriented in the Nematic Phase of ZLI-1 167

\begin{tabular}{|c|c|c|c|c|c|c|c|}
\hline Parameter & Value (Hz) & Parameter & Value (Hz) & Parameter & Value (Hz) & Parameter & Value $(\mathrm{Hz})$ \\
\hline$D_{12+13}$ & $121.04 \pm 0.05$ & $\mathrm{D}_{29+39}$ & $807.34 \pm 0.11$ & $\mathrm{~J}_{12}$ & 18.16 & $\mathrm{~J}_{210}$ & 6.71 \\
\hline $\mathrm{D}_{14}$ & $25.20 \pm 0.08$ & $\mathrm{D}_{210+310}$ & $55.18 \pm 0.09$ & $\mathrm{~J}_{13}$ & 6.64 & $\mathrm{~J}_{34}$ & 7.57 \\
\hline $\mathrm{D}_{17}$ & $190.78 \pm 0.25$ & $\mathrm{D}_{47}$ & $50.42 \pm 0.27$ & $\mathrm{~J}_{14}$ & 2.61 & $\mathrm{~J}_{35}$ & 1.45 \\
\hline $\mathrm{D}_{18}$ & $43.83 \pm 0.11$ & $\mathrm{D}_{48}$ & $63.16 \pm 0.13$ & $\mathrm{~J}_{17}$ & 153.78 & $\mathrm{~J}_{37}$ & 8.41 \\
\hline $\mathrm{D}_{19}$ & $15.36 \pm 0.14$ & $\mathrm{D}_{49}^{40}$ & $195.35 \pm 0.17$ & $\mathrm{~J}_{18}$ & 13.80 & $\mathbf{J}_{39}$ & 164.25 \\
\hline $\mathrm{D}_{110}$ & $11.84 \pm 0.17$ & $\mathrm{D}_{410}$ & $2086.64 \pm 0.17$ & $\mathbf{J}_{19}$ & 18.37 & $\mathrm{~J}_{48}$ & 7.38 \\
\hline $\mathrm{D}_{23}$ & $730.14 \pm 0.07$ & $\mathrm{D}_{58+68}^{+10}$ & $16.42 \pm 0.08$ & $\mathrm{~J}_{110}$ & -3.84 & $\mathrm{~J}_{410}^{+0}$ & 162.84 \\
\hline $\mathrm{D}_{24+34}$ & $301.89 \pm 0.05$ & $\mathrm{D}_{59+69}$ & $17.36 \pm 0.10$ & $\mathrm{~J}_{23}$ & 7.98 & $\mathrm{~J}_{59}^{+10}$ & 7.40 \\
\hline $\mathrm{D}_{25}$ & $25.38 \pm 0.08$ & $v_{4}-\left(v_{2}+v_{3}\right) / 2$ & $-248.83 \pm 0.14$ & $\mathbf{J}_{24}$ & 1.16 & $\mathrm{~J}_{68}$ & 7.38 \\
\hline $\mathrm{D}_{26+35}$ & $9.56 \pm 0.08$ & $v_{7}-v_{8}$ & $387.77 \pm 0.18$ & $\mathrm{~J}_{25}$ & 0.50 & $\mathrm{~J}_{28}$ & 164.82 \\
\hline $\mathrm{D}_{27+37}$ & $53.12 \pm 0.13$ & $v_{7}-v_{9}$ & $483.00 \pm 0.29$ & $\mathbf{J}_{26}$ & 2.04 & $\mathrm{~J}_{210}$ & 6.71 \\
\hline $\mathrm{D}_{28+38}$ & $798.56 \pm 0.09$ & $v_{7}-v_{10}$ & $378.56 \pm 0.43$ & & & & \\
\hline
\end{tabular}

Table 4 Geometrical and Order Parameters of Phenylphosphonic Dichloride Oriented in Liquid Crystal Solvent ZLI-1 167

\begin{tabular}{lccc}
\hline Parameter & Value (ZLI-1167) & Parameter & Value (ZLI-1167) \\
\hline$r_{12}$ & $2.904 \pm 0.001$ & $r_{39}$ & $1.113 \pm 0.001$ \\
$r_{14}$ & $5.710 \pm 0.003$ & $r_{410}$ & $1.110 \pm 0.000$ \\
$r_{17}$ & $1.825 \pm 0.001$ & $r_{78}$ & $1.361 \pm 0.000$ \\
$r_{23}$ & $2.498 \pm 0.001$ & $r_{89}$ & $1.417 \pm 0.000$ \\
$r_{28}$ & $1.081 \pm 0.002$ & $S_{X X}$ & $-0.0029 \pm 0.0000$ \\
$r_{34}$ & $2.507 \pm 0.000$ & $S_{Z Z}$ & $-0.095 \pm 0.0001$ \\
\hline \multicolumn{2}{c}{$r_{910}=1.395 \AA$ A (assumed). }
\end{tabular}

analysis is not different, and hence the analysis and interpretation of the dipolar couplings are not discussed in detail.

The molecule requires three elements of the ordering matrix to define its orientation and eight distance ratios to completely specify its proton skeleton. The dipolar couplings have been estimated assuming the hexagonal and pentagonal geometry for the six- and five-membered rings, respectively. Dipolar couplings and isotropic chemical shifts have been varied iteratively to analyze the spectrum.

The analysis provided 15 independent dipolar couplings. Therefore, the system is overdetermined by four dipolar couplings. Fixing the coordinates of two protons for scaling, the three-order parameters and eight coordinates of the remaining four protons have been determined iteratively. The spectral and structural parameters are reproduced in Tables 5 and 6 . It should be noted that the structural parameters have not been corrected for harmonic vibrations.

\section{METHODS TO AID THE ANALYSIS}

As mentioned earlier, analysis of spectra with more than seven or eight interacting spins is at times a formidable task because of their complexity. Several techniques have been and are being developed to aid in analysis of the complex spectrum. Some of them are multiple quantum (MQ) spectroscopy (50-57), multipulse sequences (58-61), spin decoupling $(62,63)$, specific deuteration (64), spin-echo techniques $(65,66)$, the modified 2D Z-COSY technique (67), near-magic angle spinning $(68,69)$, two-dimensional proton detected local field techniques $(70,71),{ }^{13} \mathrm{C}$-twodimensional separated local field combined with off-magic angle spinning techniques, (72-76) and the three-dimensional proton detected local field technique (77). However, each of the developed techniques has some practical difficulties, and hence so far none has been used as a routine method for the determination of molecular geometry. More advances in the field are on the way. The availability of ultrahigh-field magnetic fields (gigahertz) and the sensitivity enhancement in the modern spectrometers provide a bright future for the technique. High magnetic fields can cause a small degree of alignment of molecules in isotropic solution owing to anisotropic magnetic susceptibility $(78-83)$. This takes care of the difficulties encountered in analyses of the complex spectra of oriented systems. Another method that 

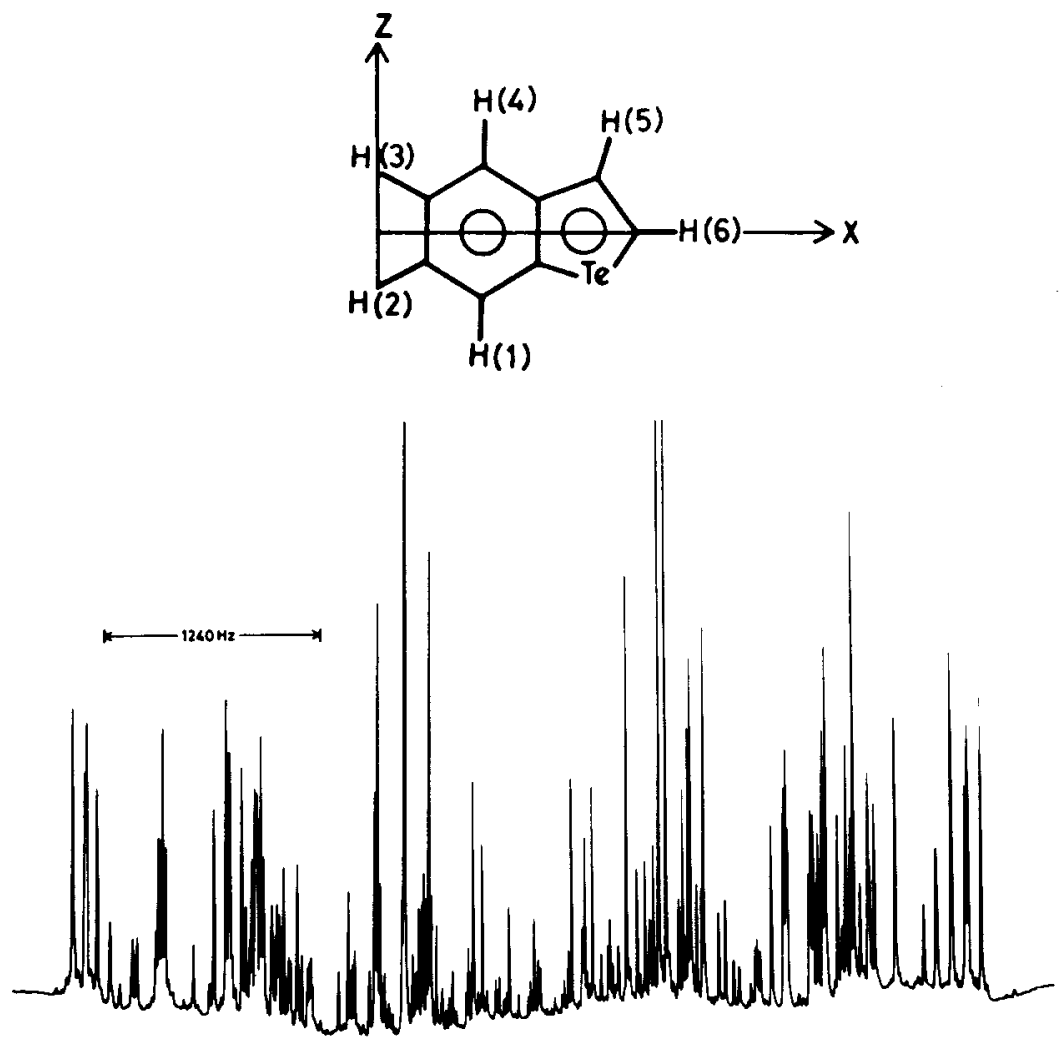

Figure 12 Structure and numbering of the molecule benzo(b) tellurophene along with the Cartesian coordinate system chosen and its proton NMR spectra at $270 \mathrm{MHz}$, oriented in the liquid crystal ZLI-1167.

Table 5 Spectral Parameters in Benzo(b)tellurophene Oriented in the Nematic Phase of ZLI-1 167

\begin{tabular}{lrcr}
\hline Parameter & \multicolumn{1}{c}{ Value $(\mathrm{Hz})$} & Parameter & \multicolumn{1}{c}{ Value $(\mathrm{Hz})$} \\
\hline$D_{12}$ & $1042.66 \pm 0.67$ & $D_{35}$ & $127.03 \pm 0.14$ \\
$D_{13}$ & $110.98 \pm 0.14$ & $D_{36}$ & $55.58 \pm 0.07$ \\
$D_{14}$ & $23.85 \pm 0.20$ & $D_{45}$ & $957.53 \pm 0.08$ \\
$D_{15}$ & $22.28 \pm 0.20$ & $D_{46}$ & $145.58 \pm 0.17$ \\
$D_{16}$ & $50.15 \pm 0.16$ & $D_{56}$ & $867.76 \pm 0.21$ \\
$D_{23}$ & $185.03 \pm 0.14$ & $\nu_{1}-v_{2}$ & $365.58 \pm 0.30$ \\
$D_{24}$ & $49.78 \pm 0.16$ & $v_{1}-v_{3}$ & $315.41 \pm 0.22$ \\
$D_{25}$ & $42.03 \pm 0.19$ & $v_{1}-v_{4}$ & $116.53 \pm 0.24$ \\
$D_{26}$ & $39.38 \pm 0.16$ & $v_{1}-v_{5}$ & $105.04 \pm 0.24$ \\
$D_{34}$ & $708.94 \pm 0.17$ & $v_{1}-v_{6}$ & $9.40 \pm 0.22$ \\
\hline
\end{tabular}

$J$ values used $(\mathrm{Hz})$ are: $J_{12}=8.27 ; J_{13}=1.17 ; J_{14}=0.48 ; J_{15}=0.65$; $J_{23}=7.22 ; J_{24}=1.02 ; J_{34}=7.97 ; J_{45}=0.14 ; J_{56}=5.66$. All other $J$ 's are zero. 
Table 6 Structural and Orientational Parameters in Benzo(b)tellurophene Oriented in ZLI-1 167

\begin{tabular}{lccc}
\hline Parameter & Value & Parameter & Value \\
\hline$r_{12} / r_{23}$ & $0.994 \pm 0.001$ & $r_{45} / r_{23}$ & $1.050 \pm 0.001$ \\
$r_{14} / r_{23}$ & $2.009 \pm 0.001$ & $r_{56} / r_{23}$ & $1.022 \pm 0.001$ \\
$r_{15} / r_{23}$ & $2.246 \pm 0.002$ & $S_{X X}$ & $-0.1404 \pm 0.0009$ \\
$r_{16} / r_{23}$ & $2.306+0.003$ & $S_{Z Z}$ & $-0.0235 \pm 0.0000$ \\
$r_{24} / r_{23}$ & $1.748 \pm 0.002$ & $S_{X Z}$ & $0.0200 \pm 0.0003$ \\
$r_{34} / r_{23}$ & $0.996 \pm 0.001$ & & \\
\hline
\end{tabular}

Scaling distance $r_{23}=2.481 \AA$ (assumed).

has been used to aid the analysis is to deuterate and estimate the order parameters from the deuteron NMR spectrum. The order parameters thus derived are used to estimate the starting parameters for the analysis of the proton NMR spectrum. With the enhanced sensitivity of the spectrometers, the natural abundance ${ }^{2} \mathrm{H}-\mathrm{NMR}$ spectra can be employed to derive orientation information. This can be subsequently used to analyze the complicated proton NMR spectra of oriented molecules. The principles described in this article will be useful even after such techniques become routinely available.

\section{ACKNOWLEDGMENTS}

Financial support by the Council of Scientific and Industrial Research, India, in the form of a research scheme is gratefully acknowledged. Useful discussions and suggestions by Professor C. L. Khetrapal, Professor Anil Kumar, and Dr. K. V. Ramanathan are thankfully acknowledged.

\section{REFERENCES}

1. R. R. Ernst, G. Bodenhausen, and A. Wokaun, Principles of Nuclear Magnetic Resonance in One and Two Dimensions, Clarendon, Oxford, 1987.

2. G. R. Luckhurst and G. W. Gray, eds., The Molecular Physics of Liquid Crystals, Academic Press, New York, 1978.

3. E. B. Priestley, "Liquid crystal mesophases," $R C A$ Rev., 1974, 35, 81-93.

4. J. W. Emsley and J. C. Lindon, NMR Spectroscopy Using Liquid Crystal Solvents, Pergamon, New York, 1975.

5. C. L. Khetrapal, A. C. Kunwar, A. S. Tracey, and P. Diehl, "Nuclear Magnetic Resonance Studies in Lyotropic Liquid Crystals," in NMR Basic Principles and Progress, Vol. 9, P. Diehl, E. Fluck, and R. Kosfeld, eds., pp. 3-74, Springer-Verlag, Berlin, 1975.
6. G. H. Brown, J. W. Doane, and V. D. Neff, $A$ Review of the Structure and Physical Properties of Liquid Crystals, CRC Press, Cleveland, Ohio, 1971.

7. E. Lafontaine, J. P. Bayle, and J. Courtieu, "High resolution NMR in cholesteric medium: Visualization of enantiomers," J. Am. Chem. Soc., 1989, 111, 8294-8296.

8. A. Meddour, A. Haudrechy, P. Berdague, W. Picoul, Y. Langiois, and J. Courtieu, "Analysis of the diastereoselectivity of a Diels-Alder cycloaddition through ${ }^{2} \mathrm{H}$ NMR in chiral liquid crystals," Tetrahedr. Assym., 1996, 7, 2489-2492.

9. P. Lesot, D. Merlet, J. Courtieu, and J. W. Emsley, "Discrimination and analysis of the NMR spectra of enantiomers dissolved in chiral liquid crystal solvents through correlation experiments," Liquid Crystals, 1996, 21, 427-435.

10. P. Lesot, D. Merlet, A. Meddour, and J. Courtieu, "Visualization of enantiomers in a polypeptide liquid-crystal solvent through carbon-13 NMR spectroscopy," J. Chem. Soc. Faraday Trans., 1995, 91, 1371-1375.

11. G. R. Luckhurst, "Magnetic resonance studies of thermotropic liquid crystals," Mol. Cryst. Liquid Cryst., 1973, 21, 125-159.

12. P. Diehl and C. L. Khetrapal, "NMR Studies of Molecules Oriented in the Nematic Phase of Liquid Crystals," in NMR Basic Principles and Progress, Vol. 1, P. Diehl, E. Fluck, and R. Kosfeld, eds., pp. 3-93, Springer-Verlag, Berlin, 1969.

13. A. Saupe, "Kernresonanzen in kristallinen Fluessigkeiten und in Kristallin fluessigen Loesungen: Teil I," Z. Naturforsch., 1964, 19a, 161-171.

14. P. J. Wojtwociz, "Introduction to molecular theory of nematic liquid crystals," RCA Rev., 1974, 35, $105-117$.

15. P. G. de Gennes, The Physics of Liquid Crystals, Clarendon Press, Oxford, 1974, p. 24.

16. E. E. Burnell and C. A. de Lange, ${ }^{" 1} \mathrm{H}$ and ${ }^{2} \mathrm{H}$ NMR of methanes partially oriented in liquidcrystal phases: Separation of rigid and non-rigid molecule effects," J. Chem. Phys., 1982, 76, 34743479.

17. J. G. Snijders, C. A. de Lange, and E. E. Burnell, "Vibration-rotation coupling in anisotropic environments: NMR of methanes in liquid crystals," $J$. Chem. Phys., 1982, 77, 5386-5396. 
18. A. Saupe, G. Englert, A. Povh, "Determination of bond angle of $\mathrm{CH}_{3}$ groups by proton magnetic resonance in nematic liquid crystalline solutions," in Advances in Chemistry Series, No. 63: Ordered Fluids and Liquid Crystals, American Chemical Society, 1967, p. 51.

19. N. Suryaprakash, R. Ugolini, and P. Diehl, "The pyrimidine-iodine interaction: A study of complex formation by NMR of oriented molecules," Magn. Reson. Chem., 1991, 29, 1024-1027.

20. R. Christy Rani Grace and N. Suryaprakash, "Multinuclear and multidimensional NMR of oriented systems - application to m-bromochlorobenzene," Proc. Natl. Acad. Sci. India, 1996, 66( A), 237-248.

21. E. K. Foord, J. Cole, M. J. Crawford, J. W. Emsley, G. Celebre, M. Longeri, and J. C. Lindon, "An NMR study of the conformational flexibility of phenylacetate dissolved in a nematic liquid crystalline solvent," Liquid Crystals, 1995, 18, 615-621.

22. M. E. Rosen, S. P. Rucker, C. Schmidt, and A. Pines, "Two-dimensional proton NMR studies of the conformations and orientations of $n$-alkanes in a liquid crystal solvent," J. Phys. Chem., 1993, 97, 3858-3866.

23. G. Celebre, G. De Luca, M. Longeri, D. Catalano, M. Lumetti, and J. W. Emsley, "An investigation of the conformation of 4-chloroethylbenzene as a solute in a nematic liquid crystalline solvent," Mol. Phys., 1995, 85, 221-231.

24. J. W. Emsley, M. I. C. Furby, and G. De Luca, "The conformation of phenyl benzoate dissolved in a nematic liquid crystalline solvent," Liquid Crystals, 1996, 21, 877-883.

25. P. Diehl, H. Kellerhals, and E. Lustig, "Computer Assistance in the Analysis of High Resolution NMR Spectra," in NMR Basic Principles and Progress, Vol. 6, P. Diehl, E. Fluck, and R. Kosfeld, eds., pp. 4-91, Springer-Verlag, Berlin, 1972.

26. XSIM Program Package, (c) 1997, Kirk Marat, Department of Chemistry, University of Manitoba, Winnipeg, Manitoba, Canada.

27. D. S. Stepehenson and G. Binsch, "Automated analysis of high-resolution NMR spectra-III - the nematic phase spectra of three allyl halides," Organ. Magn. Reson., 1980, 14, 226-233.

28. D. S. Stephenson and G. Binsch, "The molecular structure of cyclopentane in solution as obtained from a nematic phase, proton NMR study," Mol. Phys., 1980, 43, 697-710.

29. D. S. Stephenson and G. Binsch, "Automated analysis of high-resolution NMR spectra. I. Principles and computational strategy," J. Magn. Reson., 1980, 37, 395-407.

30. V. Zinn, A. Ilyasov, H. Thiele, G. Hagele, and U. Weber, "WIN-DAISY: Application to oriented molecules, analysis and simulation of NEMA-NMR spectra," Appl. Magn. Reson., 1995, 8, 311-317.

31. R. Laatikainen, M. Niemitz, U. Weber, J. Sundelin, T. Hassinen, and J. Vespäläinen, "General strate- gies for total-lineshape-type spectral analysis of NMR spectra using integral-transform iterator," $J$. Magn. Reson., 1996, 120A, 1-10.

32. C. L. Khetrapal and A. C. Kunwar, "Average orientation of molecules dissolved in nematic liquid crystals of opposite diamagnetic anisotropies," Mol. Crystals Liquid Crystals, 1981, 72, 13-16.

33. C. L. Khetrapal and A. C. Kunwar, "Determination of chemical shift anisotropy without a reference and of direct and indirect spin-spin couplings between heteronuclei," Chem. Phys. Lett., 1981, 82, 170-171.

34. P. Diehl, P. M. Henrichs, and W. Niederberger, "A study of the molecular structure and barrier to methyl rotation in $o$-chlorotoluene partially oriented in the nematic phase," Mol. Phys., 1971, 20, 139-145.

35. P. Diehl and W. Niederberger, "The vibrationally averaged molecular structure: A comparison of NMR data for oriented benzene with results from electron diffraction and Raman spectroscopy," $J$. Magn. Reson., 1973, 9, 495-502.

36. J. G. Snijders, C. A. de Lange, and E. E. Burnell, "Vibration-rotation coupling in anisotropic environment. II. Quadrupolar couplings of methanes in liquid crystals," J. Chem. Phys., 1983, 79, 29642974.

37. G. N. Patey, E. E. Burnell, J. G. Snijders, and C. A. de Lange, "Molecular solutes in nematic liquid crystals: Orientational order and electric field gradient," Chem. Phys. Lett., 1983, 99, 271-274.

38. J. B. S. Barnhoorn and C. A. de Lange, ${ }^{\text {" } 1} \mathrm{H},{ }^{19} \mathrm{~F}$ and ${ }^{2} \mathrm{H}$ NMR of monofluoro methane and deuterated analogues partially oriented in nematic liquid crystals," Mol. Phys., 1996, 88, 1-20.

39. S. Sykora, J. Vogt, H. Bosiger, and P. Diehl, "Vibrational corrections in NMR spectra of oriented molecules," J. Magn. Reson., 1979, 36, 53-60.

40. A. Saupe, "Das Protoneresonanzspektrum von orientiertem Benzol in nematisch-kristillinflussiger Losung," Zeitschrift Fur Naturforschung, 1965, 20a, $572-580$.

41. A. Saupe, "Recent results in the field of liquid crystals," Angew. Chem. Int. Edn., 1968, 7, 97-111.

42. P. J. Black, K. D. Lawson, and T. J. Flautt, "Proton magnetic resonance spectrum of benzene oriented in a lyotropic mesophase," J. Chem. Phys., 1969, 50, 542-543.

43. E. Sackmann, S. Meiboom, and L. C. Snyder, "The relation of nematic to cholesteric mesophases," $J$. Am. Chem. Soc., 1967, 89, 5981-5982.

44. L. C. Snyder and E. W. Anderson, "Analysis of the proton nuclear magnetic resonance spectrum of benzene in a nematic liquid crystal," J. Am. Chem. Soc., 1964, 86, 5023-5024.

45. P. Diehl, H. Bosiger, and H. Zimmermann, "The $r_{\alpha}$-structure of $\left[1-{ }^{13} \mathrm{C}\right]$ benzene by NMR of oriented molecules: A study of the possible precision and of solvent effects," J. Magn. Reson., 1979, 33, 113-126. 
46. J. Lounila and P. Diehl, "The effects of the correlation between vibration and rotation of partially oriented molecules on the NMR parameters: An application of the theory to the structure determination of benzene," Mol. Phys., 1984, 52, 827-845.

47. J. Jokisaari and Y. Hiltunen, "Solute molecular structure determination by NMR: Methyl iodide and benzene in thermotropic liquid crystal mixtures with ${ }^{13} \mathrm{C}$-methane as a deformation and chemical shift reference," Mol. Phys., 1983, 50, 1013-1023.

48. G. A. Nagana Gowda, S. Vivekanandan, N. Suryaprakash, and C. L. Khetrapal, "Multinuclear magnetic resonance studies of phenylphosphonic dichloride oriented in nematic phases," J. Magn. Reson., 1996, A118, 33-38.

49. N. Suryaprakash, "Application of bond polarization hypothesis to rigid ring systems: The structure of benzo(b) tellurophene oriented in the nematic phase," Proc. Natl. Acad. Sci. India, 1996, 66 $(A)$, 203-207.

50. L. D. Field, G. K. Pierens, K. J. Cross, and M. L. Terry, "Multiple quantum NMR spectroscopy of molecules aligned in liquid crystalline solvents," $J$. Magn. Reson., 1992, 97, 451-465.

51. G. K. Pierens, T. A. Carpenter, Z. D. Colebrook, L. D. Field, and L. D. Hall, Selection of multiple quantum of molecules in liquid crystalline solution using pulsed magnetic field gradients," J. Magn. Reson., 1992, 99, 398-402.

52. J. M. Polson and E. E. Burnell, "Multiple-Quantum ${ }^{1} \mathrm{H}$ NMR study of partially oriented biphenylene," J. Magn. Reson., 1994, A106, 223-228.

53. J. C. T. Rendell and E. E. Burnell, "Frequencyselective excitation in multiple quantum NMR," $J$. Magn. Reson., 1995, A112, 1-6.

54. G. Celebre, F. Castiglione, M. Longeri, and J. W. Emsley, "The NMR spectra of samples dissolved in liquid crystalline phases: Automatic analysis with the aid of multiple-quantum spectra," J. Magn. Reson., 1996, A121, 139-146.

55. T. Chandrakumar, J. M. Polson, and E. E. Burnell, "A multiple-quantum ${ }^{1} \mathrm{H}$ NMR study of conformational biasing of biphenyl in a nematic liquid crystal," J. Magn. Reson., 1996, A118, 264-271.

56. J. M. Polson and E. E. Burnell, "Conformational and orientational ordering: ${ }^{1} \mathrm{H}$ nuclear magnetic resonance of butane in a nematic liquid crystal," $J$. Chem. Phys., 1995, 103, 6891-6902.

57. D. Sandstrom and M. H. Levitt, "Structure and molecular ordering of a nematic liquid crystal studied by natural-abundance double-quantum ${ }^{13} \mathrm{C}$ NMR," J. Am. Chem. Soc., 1996, 118, 6966-6974.

58. L. Frydman, P. C. Rossomando, and B. Frydman, "Applications of multiple-pulse sequence to the simplification of liquid-crystalline NMR spectra," J. Magn. Reson., 1991, 95, 484-494.
59. P. Lesot, J. M. Ouvrard, B. N. Ouvrard, and J. Courtieu, "Coherent reduction of dipolar interactions in molecules dissolved in anisotropic media using a new multi-pulse sequence in a COSY experiment," J. Magn. Reson., 1994, 107A, 141-150.

60. D. Nanz, M. Ernst, M. Hong, M. A. Ziegeweid, K. Schmidt-Rohr, and A. Pines, "Low-power decoupling sequences for High Resolution chemical shift and local field NMR spectra of liquid crystals," $J$. Magn. Reson., 1995, A113, 169-176.

61. T. O. Levante, T. Bremi, and R. R. Ernst, "Pulsesequence optimization with analytical derivatives: Application to deuterium decoupling in oriented phases," J. Magn. Reson., 1996, A121, 167-177.

62. R. C. Hewitt, S. Meiboom, and L. C. Snyder, "Proton NMR in nematic liquid crystalline solvents: The use of deuterium decoupling," J. Chem. Phys., 1973, 58, 5089-5095.

63. J. W. Emsley, J. C. Lindon, and J. M. Tabony, "Double resonance nuclear magnetic resonance spectra of partially aligned molecules," J. Chem. Soc. Faraday Trans. II, 1973, 69, 10-22.

64. L. C. Snyder and S. Meiboom, "Theory of proton NMR with deuteron decoupling in nematic liquid crystalline solvent," J. Chem. Phys., 1973, 58, 5096-5103.

65. J. W. Emsley and D. L. Turner, "Simplification of deuterium spectra of liquid crystals by the refocussing of proton-deuterium dipolar couplings," $J$. Chem. Soc. Faraday Trans. II, 1981, 77, 1493-1508.

66. A. Kumar and C. L. Khetrapal, "Two-dimensional NMR: Application to 'oriented' molecules," $J$. Magn. Reson., 1978, 30, 137-138.

67. R. C. R. Grace and A. Kumar, "Flip-angle dependence of non-equilibrium states yielding information on connectivity of transitions and energy levels of oriented molecules," J. Magn. Reson., 1992, 99, 81-92.

68. J. Courtieu, D. W. Alderman, D. M. Grant, and J. P. Bayle, "Director dynamics and NMR applications of nematic liquid crystals, spinning at various angles from the magnetic field," J. Chem. Phys., 1982, 77, 723-730.

69. A. Kimura, N. Kuni, and H. Fujiwara, "Orientation and conformation of met-enkephalin in a liquid crystal as studied by magic-angle and near-magicangle spinning two dimensional NMR spectroscopy," J. Phys. Chem., 1996, 100, 14056-14061.

70. S. Caldarelli, M. Hong, L. Emsley, and A. Pines, "Measurement of carbon-proton dipolar couplings in liquid crystals by local dipolar field NMR spectroscopy," J. Phys. Chem., 1996, 100, 18696-18701.

71. M. Hong, A. Pines, and S. Caldarelli, "Measurement and assignment of long-range $\mathrm{C}-\mathrm{H}$ dipolar couplings in liquid crystals by two-dimensional NMR spectroscopy," J. Phys. Chem., 1996, 100, 14815-14822.

72. B. M. Fung, J. Afzal, T. L. Foss, and M. H. Chau, "Nematic ordering of 4- $n$-alkyl-4'-cyanobiphenyls 
studied by carbon-13 NMR with off-magic angle spinning," J. Chem. Phys., 1986, 85, 4808.

73. J. Courtieu, J. P. Bayle, and B. M. Fung, Progr. NMR Spectrosc., 1994, 26, 141.

74. F. Perez, J. P. Bayle, and B. M. Fung, "Orientational ordering of laterally dialkoxy branched nematics studied by one and two dimensional C-13 NMR," N. J. Chem., 1996, 20, 537-544.

75. T. Bräuniger and B. M. Fung, "Relation between the orientational ordering and the tricritical behaviour for smectic-A to smectic-C phase transition," J. Chem. Phys., 1995, 102, 7714-7720.

76. C. W. Cross and B. M. Fung, "Tricritical points of smectic A to nematic phase transitions for binary liquid crystal mixtures containing cyanobiphenyls," Liquid Crystals, 1995, 19, 863-869.

77. S. Caldarelli, A. Lesage, and L. Emsley, "Longrange dipolar couplings in liquid crystals measured by three-dimensional NMR spectroscopy," J. Am. Chem. Soc., 1996, 118, 12224-12225.

78. N. Tjandra and Ad Bax, "Measurement of Dipolar Contributions to ${ }^{1} \mathrm{~J}_{\mathrm{CH}}$ splittings from magnetic-field dependence of $\mathbf{J}$ modulation in two-dimensional NMR spectra," J. Magn. Reson., 1997, 124, 512515.

79. J. R. Tolman, J. M. Flanagan, M. A. Kennedy, and J. H. Prestegard, "NMR evidence of slow collective motions in cyanometmyoglobin," Nat. Struct. Biol., 1997, 4, 292-297.

80. J. R. Tolman, J. M. Flanagan, M. A. Kennedy, and J. H. Prestegard, "Nuclear magnetic dipole interactions in field-oriented proteins: Information for structure determination in solution," Proc. Natl. Acad. Sci., USA, 1995, 92, 9279-9283.
81. N. Tjandra, S. Grzesiek, and Ad Bax, "Magnetic field dependence of nitrogen-proton $\mathrm{J}$ splittings in $15 \mathrm{~N}$-enriched human ubiquitin resulting from relaxation interference and residual dipolar coupling," J. Am. Chem. Soc., 1996, 118, 6264-6272.

82. A. A. Bothner-By, C. Gayathri, P. C. M. van Zijl, C. MacLean, J. Ji Lai, and K. M. Smith, "High-field orientation effects in the high-resolution proton NMR spectra of diverse porphyrins," Magn. Reson. Chem., 1985, 23, 935-938.

83. C. Gayathri, A. A. Bothner-By, P. C. M. van Zijl, and C. MacLean, "Dipolar magnetic field effects in NMR spectra of liquids," Chem. Phys. Lett., 1982, 87, 192-196.

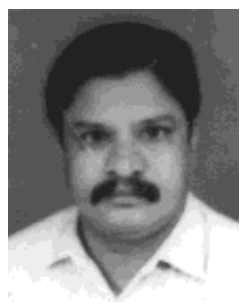

N. Suryaprakash holds B.Sc. and M.Sc. degrees (1975 and 1977) from the University of Mysore, and a Ph.D. degree (1986) from the University of Bangalore, Karnataka, India. The research field of NMR spectroscopy of oriented molecules was introduced to him by Professor C. L. Khetrapal and Dr. A. C. Kunwar. He has nearly 50 publications. His research interests involve the elucidation of molecular structure, the dynamics of liquid crystal director, and developments of new techniques in one- and two-dimensional NMR. He is the recipient of the Bruker Young NMR Scientist award for his outstanding contributions in the field of NMR spectroscopy and its applications. $\mathrm{He}$ is also the recipient of the Young Scientist award of the Karnataka Association for the Advancement of Science for his outstanding contributions in the field of chemical sciences. Presently, he holds a faculty position at Sophisticated Instruments Facility, Indian Institute of Science, Bangalore, India. 